\title{
Dynamic Time-Frequency Division Duplex
}

\author{
Mohsen Mohammadkhani Razlighi, Student Member, IEEE, Nikola Zlatanov, Member, IEEE, and Petar Popovski, \\ Fellow, IEEE
}

\begin{abstract}
In this paper, we introduce dynamic time-frequencydivision duplex (D-TFDD), which is a novel duplexing scheme that combines time-division duplex (TDD) and frequency-division duplex (FDD). In D-TFDD, a user receives from the base station (BS) on the downlink in one frequency band and transmits to the BS on the uplink in another frequency band, as in FDD. Next, the user shares its uplink transmission (downlink reception) on the corresponding frequency band with the uplink transmission or the downlink reception of another user in a D-TDD fashion. Hence, in a given frequency band, the BS communicates with user 1 (U1) and user 2 (U2) in a D-TDD fashion. The proposed D-TFDD scheme does not require inter-cell interference (ICI) knowledge and only requires channel state information (CSI) of the local BS-U1 and BS-U2 channels. Thereby, it is practical for implementation. The proposed D-TFDD scheme increases the throughput region between the $\mathrm{BS}$ and the two users in a given frequency band, and significantly decreases the outage probabilities on the corresponding BS-U1 and BS-U2 channels. Most importantly, the proposed D-TFDD scheme doubles the diversity gain on both the corresponding BS-U1 and the BS-U2 channels compared to the diversity gain of existing duplexing schemes, which results in very large performance gains.
\end{abstract}

\section{INTRODUCTION}

Traditionally, a half-duplex (HD) base station (BS) operates in either the time-division duplex (TDD) mode or the frequency-division duplex (FDD) mode in order to receive and transmit information from/to its users. In the TDD mode, a user uses the same frequency band for uplink and downlink, while uplink and downlink transmissions occur in different time slots [2], see Fig. 1] On the other hand, in the FDD mode, a user is allocated two frequency bands, one dedicated for uplink and the other one for downlink, where the uplink and downlink transmissions occur simultaneously [2], see Fig. 1] In this paper, we introduce time-frequency-division duplex (TFDD), which is a novel duplexing scheme that combines TDD and FDD, and yields significant performance gains compared to TDD and FDD.

\section{A. Background on the Different Types of Duplexing Schemes}

1) Static vs. Dynamic Duplexing: In general, the duplexing method between a BS and its users can be static or dynamic.

This work has been published in part at IEEE ICC 2018 [1].

This work was supported by the Australian Research Council Discovery Project under Grant DP180101205.

Content presented in this article is subjected to Australian Provisional Patent Application 2019903224.

M. M. Razlighi N. Zlatanov are with the Department of Electrical and Computer Systems Engineering, Monash University, Melbourne, VIC 3800, Australia (e-mails: mohsen.mohammadkhanirazlighi@monash.edu and nikola.zlatanov@monash.edu.)

P. Popovski is with the Department of Electronic Systems, Aalborg University, Denmark (e-mail: petarp@es.aau.dk).
In static duplexing, the time-frequency resources in which the BS performs uplink receptions and downlink transmissions from/to the user are prefixed and unchangeable over time [3]. On the other hand, in dynamic duplexing schemes, each time-frequency resource unit can be dynamically allocated for communications based on the instantaneous channel state information (CSI). As a result, dynamic duplexing schemes achieve a much better performance compared to static duplexing schemes [4], [5], and thereby have attracted significant research interest [4]-[8].

2) Centralized Dynamic Duplexing vs. Distributed Dynamic Duplexing: A dynamic duplexing scheme can be implemented in either centralized or distributed fashion [6]. In centralized dynamic duplexing schemes, the decision for allocating the time-frequency resources for communication is performed at a central node, which then informs all BSs about the decision. In this way, the communication between neighbouring cells can be synchronized in order to minimize inter-cell interference 1 (ICI) [7]-[17]. However, centralized dynamic duplexing schemes require at the central node full CSI from all links in all cells in order for the central node to make an optimal decision for allocating the time-frequency resources for each BS. In addition, the central node also needs to inform all other network nodes about the scheduling decisions. This requires a large amount of signalling information to be exchanged between the central node and all other network nodes. As a result, implementation of centralized dynamic duplexing schemes, in most cases, is infeasible in practice.

On the other hand, in distributed dynamic duplexing schemes, each BS allocates the time-frequency resources for its users without any synchronization with other BSs [13], [18], [19]. To this end, only local CSI is needed at each BS. As a result, distributed dynamic duplexing schemes are much more appropriate for practical implementation compared to the centralized dynamic duplexing scheme. However, distributed dynamic duplexing schemes have to cope with higher ICI than centralized dynamic duplexing schemes.

The proposed TFDD scheme can be characterized as a distributed dynamic duplexing scheme, which means it is suitable for practical implementation.

\section{B. Contribution}

In this paper, we introduce dynamic time-frequency-division duplex (D-TFDD), which is a novel duplexing scheme that combines D-TDD and FDD. In D-TFDD, a user receives from the BS on the downlink in one frequency band and transmits to the BS on the uplink in another frequency band,

\footnotetext{
${ }^{1} \mathrm{ICI}$ emerges when BSs and users in neighbouring cells transmit and receive on the same frequency band.
} 


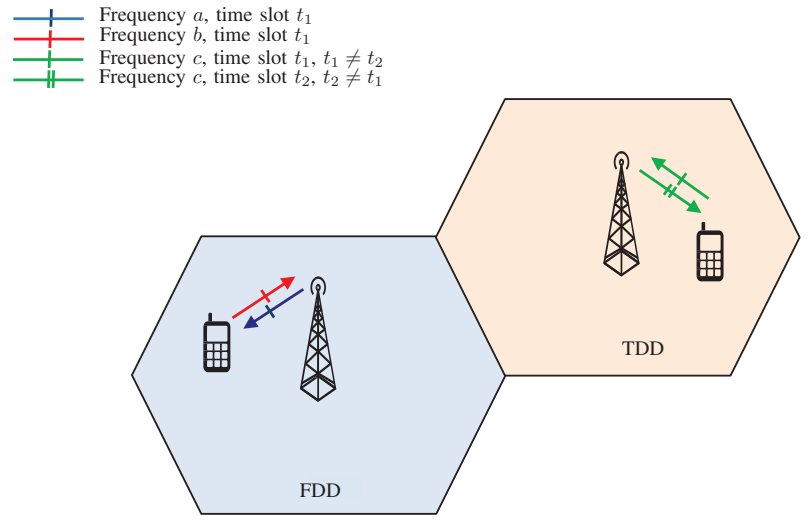

Fig. 1. System model of networks with FDD and TDD Communication.

as in FDD. Next, the user shares its uplink transmission (downlink reception) on the corresponding frequency band with the uplink transmission or the downlink reception of another user in a D-TDD fashion. Hence, in a given frequency band, the BS communicates with user 1 (U1) and user 2 (U2) in a D-TDD fashion. The proposed D-TFDD scheme does not require ICI knowledge and only requires CSI of the local BS-U1 and BS-U2 channels. Thereby, it is practical for implementation. The proposed D-TFDD scheme increases the throughput region between the BS and the two users in a given frequency band, and significantly decreases the outage probabilities on the corresponding BS-U1 and BS-U2 channels. Most importantly, the proposed D-TFDD scheme doubles the diversity gain on both the corresponding BS$\mathrm{U} 1$ and the BS-U2 channels compared to the diversity gain of existing duplexing schemes, which results in very large performance gains.

\section{Relevance of D-TFDD to $5 G$ and Beyond}

One of the prominent aspects of fifth generation $(5 \mathrm{G})$ mobile networks is having a flexible physical layer design. In one hand, this capability facilitates implementing challenging physical layer protocols, and on the other hand opens the door for unconventional schemes to be implemented on the physical layer. Such a flexible hardware-software design can easily accommodate our D-TFDD scheme and thereby improve the performance of $5 \mathrm{G}$ networks [20]. In addition, distributed resource allocation for dense heterogeneous wireless networks is in one of the main scopes of 5G [20], which also fits well with our D-TFDD scheme. Moreover, proposed scheme is applicable to multi-tier multi-cell systems, which is another feature of $5 \mathrm{G}$ networks.

\section{Related Works on D-TDD and D-FDD}

Distributed D-TDD schemes for BSs have been investigated in [18], [19], [21]-[25] and references therein. In particular, [18] proposed cooperation among cells that resembles a centralized D-TDD scheme. The authors in [19] proposed a DTDD scheme which alleviates the ICI impairment by splitting the uplink and downlink frequency. Authors in [21] and [22] investigate a distributed D-TDD scheme designed for multipleantennas. The work in [23] proposes a distributed multi-user D-TDD scheduling scheme, where the ICI is not taken into account, which may lead to poor performance in practice. The authors in [24] investigated an identical network as in [23], but with ICI taken into account. However, the solution in [24] is based on a brute-force search algorithm for allocating the time slots. Authors in [25] proposed a D-TDD scheme that performs optimal power, rate, and user allocation. However, the ICI level in [25] is assumed to be fixed during all time slots, which may not be an accurate model of ICI in practice, since due to the fading and the power-allocations at neighbouring BSs, the ICI varies with time.

On the other hand, D-FDD has been introduced in [13], where the authors proposed a scheme for adapting the downlink to uplink bandwidth ratio.

We note that [13], [18], [19], [21]-[25] require full knowledge of the ICI, which may not be practical, as discussed in Sec. II-D. We also note that the schemes in [13], [18], [19], [21]-[25] transform to the static-TDD and/or static-FDD scheme when ICI is not known. Hence, the static-TDD and/or static-FDD are much more practical for implementation than the D-FDD duplexing scheme since they do not require ICI knowledge.

The rest of this paper is organized as follows. In Section III we present the system and channel model. In Sections III and IV, we present the D-TFDD schemes for the cases when the ICI is known and unknown, respectively. Simulation and numerical results are provided in Section $\mathrm{V}$ and the conclusions are drawn in Section VI.

\section{System AND Channel Model}

In the following, we consider a cellular network consisting of cells, where each cell has a single BS and users that the BS serves.

\section{A. Frequency and Time Allocation in D-TFDD}

In the proposed D-TFDD scheme, we have two possible frequency allocation schemes at each BS, Frequency Allocation Scheme 1 shown in Fig. 2 and Frequency Allocation Scheme 2 shown in Fig. 3 In both frequency allocation schemes, each user is allocated two distinct frequency bands whithin the cell, one for uplink transmission and the other for downlink reception, identical as in FDD, see Figs. 2 and 3 In Frequency Allocation Scheme 1, the frequency band of a user allocated for uplink transmission (downlink reception) is shared in a D-TDD fashion with the uplink transmission (downlink reception) of another user, as shown in Figs. 2. Whereas, in Frequency Allocation Scheme 2, the frequency band of a user allocated for uplink transmission (downlink reception) is shared in a D-TDD fashion with the downlink reception (uplink transmission) of another user, as shown in Figs. 3. Hence, for $N$ users, the BS needs to allocate $N$ frequency bands in D-TFDD, same as in TDD.

Frequency Allocation Scheme 1 is more appropriate for cellular communication networks, where the transmit powers of BSs are much higher than the transmit powers of the 


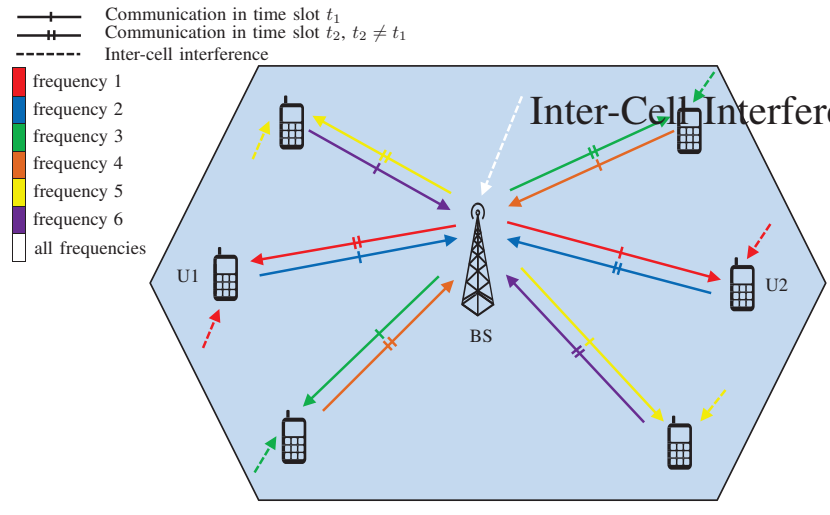

Fig. 2. Frequency Allocation Scheme 1.

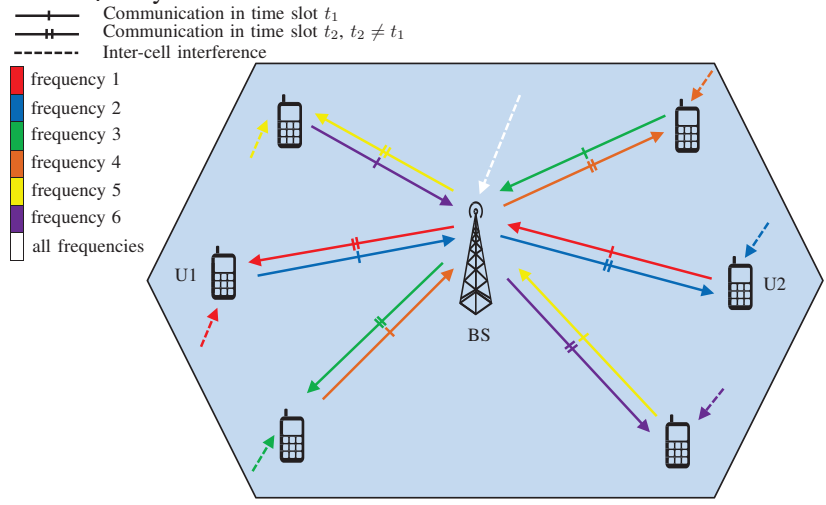

Fig. 3. Frequency Allocation Scheme 2.

users. Specifically, when Frequency Allocation Scheme 1 is applied to every BS in the cellular network such that the uplink frequency bands are used only for uplink and the downlink frequency bands are used only for downlink at all BSs, then all uplink links receive inter-cell interference only from other uplink transmissions and all downlink links receive inter-cell interference only from other downlink transmissions. As a result, the problem in existing D-TDD schemes of strong downlink transmission from one BS interfering with the weak uplink reception at another BS is avoided in DTFDD with Frequency Allocation Scheme 1. On the other hand, Frequency Allocation Scheme 2 might be more suitable for networks where the uplink and downlink transmissions have equal powers.

\section{B. The Three-Node Subnetwork}

We assume that there is no interference between different frequency bands. The only interference at a user/BS in a given frequency band is a result of the transmission in the same frequency band from the users and the BSs in other cells. As a result, for a given frequency band, the considered cellular network employing the D-TFDD scheme can be divided into three-node subnetworks, where each subnetwork consists of a BS and two users working in the same frequency band that are impaired by ICI coming from the rest of the subnetworks working in the same frequency band, as shown in Fig. 4 . Depending on whether the downlink reception (uplink transmission) in a given frequency band is shared with the downlink reception or the uplink transmission of another user, there can

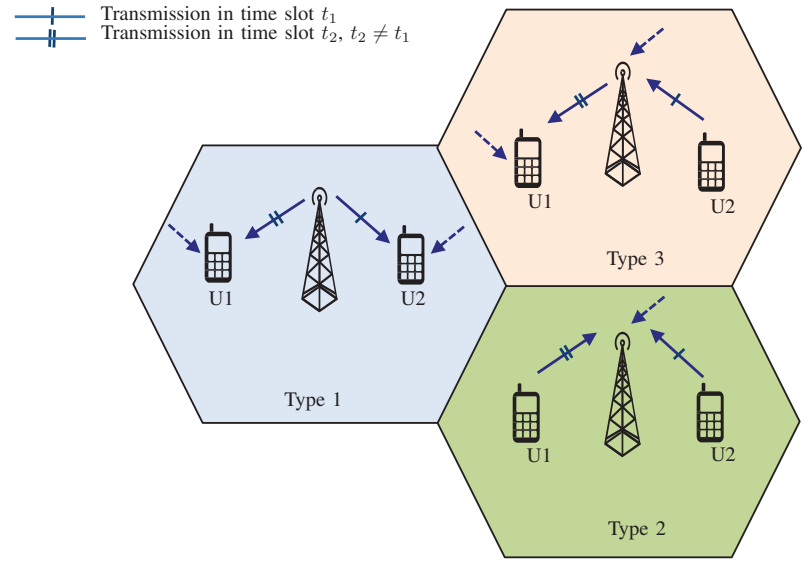

Fig. 4. System model of a BS and two users employing D-TFDD on a specific frequency band for Type 1, 2 and 3.

be three types of three-node subnetworks, as shown in Fig. 4 . Type 1 is when both users perform downlink receptions in the given frequency band. Type 2 is when both users perform uplink transmissions in the given frequency band. And Type 3 is when one of the users performs uplink transmission and the other user performs downlink reception in the given frequency band. Note that the three types of three-node subnetworks differ only in the direction of the transmission on the BS-U1 and BS-U2 channels.

Now, in order for the D-TFDD to be a distributed duplexing scheme, each of the three-node subnetworks must perform D-TFDD independently from the rest of the subnetworks in the cellular network. As a result, without loss of generality, the equivalent system model that needs to be investigated is comprised of a BS communicating with $\mathrm{U} 1$ and $\mathrm{U} 2$ in different time slots but in the same frequency band, where receivers are impaired by ICI, as shown in Fig. 4

Remark 1: The considered three-node subnetworks shown in Fig. 4 can also represent the decoupled access [26], [27] by BS and users switching places, where a user is connected to two BSs and performs uplink-transmission or downlinkreception to/from BS1 and uplink-transmission or downlinkreception to/from BS2 in the same frequency band. Since the decoupled system can be obtained by the proposed system where BS and users switch places, the proposed D-TFDD scheme is also applicable to the decoupled access.

\section{Inter-Cell Interference}

The receiving nodes of a given three-node subnetwork are impaired by interference from all other nodes in the network that transmit on the frequency band used for reception at the BS, and/or U1, and/or U2, also referred to as ICI, see Fig. 4. Let the power of the ICI at the receiving nodes on the BS-U1 and BS-U2 channels in time slot $t$ be denoted by $\gamma_{I 1}(t)$ and

\footnotetext{
${ }^{2}$ Time slot is a time interval that is equal or smaller than the duration of the coherence interval. Moreover, we assume that a time slot is long enough such that a capacity achieving codeword can be transmitted during one time slot.

${ }^{3}$ The subscripts 1 and 2 are used to symbolize the BS-U1 and the BS-U2 channels, respectively.
} 
$\gamma_{I 2}(t)$, respectively. Then, we can obtain $\gamma_{I 1}(t)$ and $\gamma_{I 2}(t)$ as

$$
\begin{aligned}
& \gamma_{I 1}(t)=\sum_{k \in \mathcal{K}} P_{k} \gamma_{k 1}(t), \\
& \gamma_{I 2}(t)=\sum_{k \in \mathcal{K}} P_{k} \gamma_{k 2}(t),
\end{aligned}
$$

where $\mathcal{K}$ is the set of interfering nodes, $P_{k}$ is the power of interfering node $k$, and $\gamma_{k 1}(t)$ and $\gamma_{k 2}(t)$ are the square of the channel gains between interfering node $k$ and the receiver on the BS-U1 channel, and interfering node $k$ and the receiver one the BS-U2 channel, in time slot $t$, respectively.

\section{Inter-Cell Interference Estimation Overhead}

A network comprised of $K$ HD nodes, all operating in the same frequency band, requires at least $K$ estimation periods in order for the ICI to be estimated at all $K$ nodes. To see this, note that a HD node can either receive or transmit in a given frequency band. As a result, in order for a HD node to estimate the interference from the remaining $K-1 \mathrm{HD}$ nodes, an estimation period must be dedicated for this purpose in which the considered HD node receives and the rest of the $K-1$ HD transmit. Since this process has to be repeated for each of the $K$ HD nodes, it follows that a network comprised of $K \mathrm{HD}$ nodes, all operating in the same frequency band, must dedicate $K$ time periods for ICI estimation at the $K$ HD nodes. Hence, ICI estimation at $K$ HD nodes entails an overhead of $K$ estimation periods. In addition, since the transmission schedule of the different HD nodes is not known in advance, the estimated ICI may differ significantly than the real one, which means that the overhead of $K$ time periods is a lower bound of the actual number of time periods needed for estimation of the actual ICI. In fact, this is a key point. The only realistic way to have the transmission schedule of the HD nodes known in advance is to have a central controller that gathers all the channels, makes a scheduling decision for each link in each time slot and forwards that decision to the nodes. This is not feasible in current systems and will likely not be feasible in future systems as long as the coherence time equals a time slot during which the CSI acquisition, the transmission of the scheduling decisions, and the actual transmission of data need to take place.

The overhead needed for ICI estimation requires resources that may prohibit ICI estimation in practice. As a result, in this paper, we investigate the practical case without ICI knowledge, and propose a distributed D-TFDD scheme for this practical scenario. In addition, in order to obtain an upper bound on the performance of the D-TFDD in terms of outage probability and throughput rate for unknown ICI, we will also investigate the case where the ICI is known at the nodes. Consequently, we will propose distributed D-TFDD schemes for the cases with and without ICI knowledge, and show that the proposed distributed D-TFDD scheme without ICI knowledge has performance which is close to its upper bound achieved when the ICI is known.

\footnotetext{
${ }^{4}$ For D-TFDD Type $3, \gamma_{I 1}(t)=\gamma_{I 2}(t)$.
}

Remark 2: The above method for estimating the interference works only if $K$ is known. In practice, $K$ is unknown and, in that case, estimating the interference requires even more resources.

\section{E. Channel Model}

In a given subnetwork, we assume that the BS-U1 and BSU2 channels are complex-valued additive white Gaussian noise (AWGN) channels impaired by slow fading and ICI. Next, we assume that the transmission time is divided into $T \rightarrow \infty$ time slots. Furthermore, we assume that the fading is constant during one time slot and changes from one time slot to the next. In time slot $t$, let the complex-valued fading gains of the BS-U1 and BS-U2 channels be denoted by $h_{1}(t)$ and $h_{2}(t)$, respectively. Moreover, let the variances of the complex-valued AWGNs at receiving nodes of the the BS-U1 and BS-U2 channels be denoted by $\sigma_{1}^{2}$ and $\sigma_{2}^{2}$, respectively 5 . For convenience, we define normalized magnitude-squared fading gains of the BS-U1 and BS-U2 channels as $\gamma_{1}(t)=\left|h_{1}(t)\right|^{2} / \sigma_{1}^{2}$ and $\gamma_{2}(t)=\left|h_{2}(t)\right|^{2} / \sigma_{2}^{2}$, respectively. Furthermore, let the transmit powers of the transmit nodes on the BS-U1 and BSU2 channels in time slot $t$ be denoted by $P_{1}$ and $P_{2}, \forall t$, respectively.

Using the above notation and taking into account the AWGNs and the ICIs given by (1) and (2), and treating the ICI as noise, the capacities of the BS-U1 and BS-U2 channels in time slot $t$, denoted by $C_{1}(t)$ and $C_{2}(t)$, respectively, are obtained as

$$
\begin{aligned}
& C_{1}(t)=\log _{2}\left(1+\frac{P_{1} \gamma_{1}(t)}{1+\gamma_{I 1}(t)}\right) \\
& C_{2}(t)=\log _{2}\left(1+\frac{P_{2} \gamma_{2}(t)}{1+\gamma_{I 2}(t)}\right)
\end{aligned}
$$

\section{F. Discrete-Rate Transmission}

We assume that the transmit nodes on the BS-U1 or the BS-U2 channels transmit their codewords with rates which are selected from discrete finite sets of data rates, denoted by $\mathcal{R}_{1}=\left\{R_{1}^{1}, R_{1}^{2}, \ldots, R_{1}^{M}\right\}$ and $\mathcal{R}_{2}=\left\{R_{2}^{1}, R_{2}^{2}, \ldots, R_{2}^{L}\right\}$, respectively, where $M$ and $L$ denote the total number of non-zero data rates available for transmission at the transmit nodes on the BS-U1 and BS-U2 channels, respectively. This allows us to have a transmission model used in practice which also converges to continuous transmission rates model when $M \rightarrow \infty$ and $L \rightarrow \infty$, and to the single fixed-rate transmission model when $M=1$ and $L=1$.

\section{D-TFDD FOR KNOWN ICI}

In this section, we assume that the ICI is known at the nodes at the start of each time slot. Although this assumption is not practical as discussed in Sec. II-D it will enable us to obtain an upper bound on the practical D-TFDD without ICI knowledge at the nodes.

\footnotetext{
${ }^{5}$ For D-TFDD Type $2, \sigma_{1}^{2}=\sigma_{2}^{2}$.
} 


\section{A. BS-U1 and BS-U2 Throughput Region}

In a given time slot, depending on whether we are communicating on the BS-U1 and BS-U2 channels, the considered three-node subnetwork, shown in Fig. 4 can be in one of the following three states

State 0: No transmission occurs on both BS-U1 and BS-U2 channels.

State 1: Channel BS-U1 is selected for transmission and channel BS-U2 is inactive/silent.

State 2: Channel BS-U2 is selected for transmission and channel BS-U1 is inactive/silent.

In State 1, the transmitting node on the BS-U1 channel can choose to transmit with any rate in the set $\mathcal{R}_{1}$. Similarly, in State 2, the transmitting node on the BS-U2 channel can choose to transmit with any rate in the set $\mathcal{R}_{2}$. In order to model these states for time slot $t$, we introduce the binary variables $q_{1}^{m}(t), m=1,2 \ldots, M$ and $q_{2}^{l}(t)$, for $l=1, \ldots, L$, defined as

$$
q_{1}^{m}(t)=\left\{\begin{array}{l}
1 \quad \begin{array}{l}
\text { if channel BS-U1 is selected for the } \\
\text { transmission of a codeword with rate } R_{1}^{m} \\
\text { and power } P_{1} \text { in time slot } t \\
0 \quad \begin{array}{l}
\text { otherwise, }
\end{array}
\end{array}
\end{array}\right.
$$

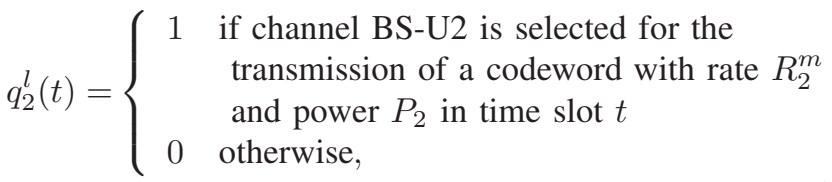

respectively. In addition, since the considered network can be in one and only one state in time slot $t$, the following has to hold

$$
\sum_{m=1}^{M} q_{1}^{m}(t)+\sum_{l=1}^{L} q_{2}^{l}(t) \in\{0,1\}
$$

where if $\sum_{m=1}^{M} q_{1}^{m}(t)+\sum_{l=1}^{L} q_{2}^{l}(t)=0$ holds, then both the BS-U1 and the BS-U2 channels are inactive in time slot $t$. Condition (7) results from the HD constraint of the BS, i.e., the BS can either receive or transmit in a given time slot on the same frequency band.

Since the available transmission rates are discrete, outages can occur. An outage is defined as the event when data rate of the transmitted codeword is larger than the capacity of the underlying channel. To model the outages on the BS-U1 and the BS-U2 channels, we introduce the following auxiliary binary variables, $O_{1}^{m}(t)$, for $m=1, \ldots, M$, and $O_{2}^{l}(t)$, for $l=1, \ldots, L$, respectively, defined as

$$
\begin{aligned}
& O_{1}^{m}(t)=\left\{\begin{array}{ll}
1 & \text { if } C_{1}(t) \geq R_{1}^{m} \\
0 & \text { if } C_{1}(t)<R_{1}^{m}
\end{array},\right. \\
& O_{2}^{l}(t)= \begin{cases}1 & \text { if } C_{2}(t) \geq R_{2}^{l} \\
0 & \text { if } C_{2}(t)<R_{2}^{l} .\end{cases}
\end{aligned}
$$

Using $O_{1}^{m}(t)$, we can obtain that in time slot $t$ a codeword transmitted on the BS-U1 channel with rate $R_{1}^{m}$ can be decoded correctly at the receiver if and only if (iff) $q_{1}^{m}(t) O_{1}^{m}(t)>0$ holds. Similarly, using $O_{2}^{l}(t)$, we can obtain that in time slot $t$ a codeword ctransmitted on the BS-U2 channel with rate $R_{2}^{l}$ can be decoded correctly at receiver iff $q_{2}^{l}(t) O_{2}^{l}(t)>0$ holds. Thereby, the average achieved throughputs during $T \rightarrow \infty$ time slots on the BS-U1 and BSU2 channels, denoted by $\bar{R}_{1}$ and $\bar{R}_{2}$, respectively, are given by

$$
\begin{aligned}
& \bar{R}_{1}=\lim _{T \rightarrow \infty} \frac{1}{T} \sum_{t=1}^{T} \sum_{m=1}^{M} R_{1}^{m} q_{1}^{m}(t) O_{1}^{m}(t), \\
& \bar{R}_{2}=\lim _{T \rightarrow \infty} \frac{1}{T} \sum_{t=1}^{T} \sum_{l=1}^{L} R_{2}^{l} q_{2}^{l}(t) O_{2}^{l}(t) .
\end{aligned}
$$

The throughput pair $\left(\bar{R}_{1}, \bar{R}_{2}\right)$, defined by 10 and (11), for some fixed vectors $\left[q_{1}^{m}(1), q_{1}^{m}(2), \ldots, q_{1}^{m}(T)\right]$ and $\left[q_{2}^{l}(1), q_{2}^{l}(2), \ldots, q_{2}^{l}(T)\right]$ gives one point on the graph where $\bar{R}_{1}$ and $\bar{R}_{2}$ are axis. All possible combinations of $\left[q_{1}^{m}(1), q_{1}^{m}(2), \ldots, q_{1}^{m}(T)\right]$ and $\left[q_{2}^{l}(1), q_{2}^{l}(2), \ldots, q_{2}^{l}(T)\right]$ give a region of points that is bounded by a maximum boundary line of the BS-U1 and BS-U2 throughput region. Our task now is to find the maximum boundary line of this BS-U1 and BS-U2 throughput region, $\left(\bar{R}_{1}, \bar{R}_{2}\right)$, by selecting the optimal values of $q_{1}^{m}(t), q_{2}^{l}(t), \forall m, l, t$, respectively.

The maximum boundary line of the BS-U1 and BS-U2 throughput region $\left(\bar{R}_{1}, \bar{R}_{2}\right)$, given by 10 and 11 , can be found from the following maximization problem

$$
\begin{aligned}
& \underset{q_{1}^{m}(t), q_{2}^{l}(t), \forall l, m, t .}{\operatorname{Maximize}:} \mu \bar{R}_{1}+(1-\mu) \bar{R}_{2} \\
& \text { Subject to : } \\
& \text { C1 : } q_{1}^{m}(t) \in\{0,1\}, \forall m \\
& \text { C2 : } q_{2}^{l}(t) \in\{0,1\}, \forall l \\
& \text { C3 }: \sum_{m=1}^{M} q_{1}^{m}(t)+\sum_{l=1}^{L} q_{2}^{l}(t) \in\{0,1\},
\end{aligned}
$$

where $\mu$ is a priori given constant which satisfies $0 \leq \mu \leq 1$. A specific value of $\mu$ provides one point on the boundary line of the BS-U1 and BS-U2 throughput region $\left(\bar{R}_{1}, \bar{R}_{2}\right)$. By varying $\mu$ from zero to one, the entire boundary line of the BS$\mathrm{U} 1$ and BS-U2 throughput region $\left(\bar{R}_{1}, \bar{R}_{2}\right)$ can be obtained. The solution of 12 is given in the following theorem.

Theorem 1: The optimal state and rate selection variables, $q_{1}^{m}(t)$ and $q_{2}^{l}(t)$, of the D-TFDD scheme for known ICI that maximize the BS-U1 and BS-U2 throughput region of the considered subnetwork, which are found as the solution of

\footnotetext{
${ }^{6}$ Note that the defined throughput region is not the capacity region.
} 
(12), are given as

BS-U1 transmission $>q_{1}^{m^{*}}(t)=1, q_{1}^{m}(t)=0, \forall m \neq m^{*}$

$$
\begin{aligned}
& \text { and } q_{2}^{l}(t)=0, \forall l \\
& \text { if }\left[\Lambda_{1}^{m^{*}}(t) \geq \Lambda_{2}^{l^{*}}(t) \text { and } \Lambda_{1}^{m^{*}}(t)>0\right],
\end{aligned}
$$

BS-U2 transmission $q_{2}^{l^{*}}(t)=1, q_{2}^{l}(t)=0, \forall l \neq l^{*}$

$$
\text { and } q_{1}^{m}(t)=0, \forall m
$$

$$
\text { if }\left[\Lambda_{2}^{l^{*}}(t)>\Lambda_{1}^{m^{*}}(t) \text { and } \Lambda_{2}^{l^{*}}(t)>0\right] \text {, }
$$

Silence $\triangleright q_{1}^{m}(t)=0, \forall m$ and $q_{2}^{l}(t)=0, \forall l$

$$
\text { if }\left[\Lambda_{1}^{m^{*}}(t)=0 \text { and } \Lambda_{2}^{l^{*}}(t)=0\right] \text {, }
$$

where $\Lambda_{1}^{m}(t), \Lambda_{2}^{l}(t), m^{*}$ and $l^{*}$ are defined as

$$
\begin{aligned}
\Lambda_{1}^{m}(t) & =\mu R_{1}^{m} O_{1}^{m}(t), \\
\Lambda_{2}^{l}(t) & =(1-\mu) R_{2}^{l} O_{2}^{l}(t), \\
m^{*} & =\arg \max _{m}\left\{\Lambda_{1}^{m}(t)\right\}, \\
l^{*} & =\arg \max _{l}\left\{\Lambda_{2}^{l}(t)\right\} .
\end{aligned}
$$

Proof: Please refer to Appendix A for the proof.

Note that for the proposed D-TFDD scheme in Theorem 1 to operate, the receivers of the BS-U1 and BS-U2 channels need to know $O_{1}^{m}(t)$ and $O_{2}^{l}(t)$, respectively, $\forall m, l$, at the start of time slot $t$, which requires knowledge of the ICI.

\section{B. Diversity Gain of the Proposed D-TFDD for Known ICI}

It is quite interesting to investigate the diversity gain achieved with the D-TFDD scheme for known ICI proposed in Theorem 1. In the literature, the asymptotic outage probability, from which the diversity gain is obtained, is derived assuming only a single available transmission rate at the transmitter, see [28]. Following this convention, in the following, we derive the asymptotic outage probabilities of the BS-U1 and the BS-U2 channels, denoted by $P_{\text {out }}$, achieved with the D-TFDD scheme for known ICI proposed in Theorem 1 for $\mu=\frac{1}{2}, M=L=1$, $P_{1}=P_{2}$, and $R_{1}^{1}=R_{2}^{1}=R_{0}$. For simplicity, we only investigate the case of Rayleigh fading, and also assume that the BS-U1 and BS-U2 channels are affected by independent and identically distributed (i.i.d) fading.

Theorem 2: The asymptotic outage probability of the DTFDD scheme for known ICI proposed in Theorem 1 for the case of Rayleigh fading and when $\mu=\frac{1}{2}, M=L=1$, $P_{1}=P_{2}=P$, and $R_{1}^{1}=R_{2}^{1}=R_{0}$ hold, is given by

$$
P_{\text {out }} \rightarrow \frac{\gamma_{\mathrm{th}}^{2} \hat{\Omega}_{I}}{\Omega_{0}^{2}}, \text { as } P \rightarrow \infty,
$$

where $\gamma_{\mathrm{th}}=\frac{2^{R_{0}}-1}{P}, \Omega_{0}=E\left\{\frac{\left|h_{1}(t)\right|^{2}}{\sigma_{1}^{2}}\right\}=E\left\{\frac{\left|h_{2}(t)\right|^{2}}{\sigma_{2}^{2}}\right\}$, and $\hat{\Omega}_{I}=E\left\{\left(1+\gamma_{I 1}(t)\right)\left(1+\gamma_{I 2}(t)\right)\right\}$.

As can be seen from (18), the outage probability $P_{\text {out }}$ has a diversity gain of two.

Proof: Please refer to Appendix B for the proof.

Note that existing D-TDD and D-FDD schemes achieve a diversity gain of one, which leads to the conclusion that the proposed D-TFDD scheme doubles the diversity gain compared to existing duplexing schemes, which in turn leads to very large performance gains, cf. Sec. V

\section{D-TFDD FOR UNKNOWN ICI}

The D-TFDD scheme proposed in Section III requires the receivers of the BS-U1 and BS-U2 channels to know $O_{1}^{m}(t)$ and $O_{2}^{l}(t)$, respectively, $\forall m, l$, at the start of time slot $t$, for $t=1,2, \ldots T$, which requires ICI knowledge. However, as discussed in Sec. II-D the estimation of the ICI entails huge cost for a cellular network comprised of $K$ HD nodes which may not be practical. Motivated by this problem, in the following, we propose a D-TFDD scheme where the nodes do not have knowledge of the ICI, and as a result, the network nodes do not have to waste huge resources for estimating the ICI. We only assume that the CSI of the BS$\mathrm{U} 1$ and BS-U2 channels are known at the BS, i.e., we assume local CSI knowledge at the BS. Specifically, the BS knows $\gamma_{1}(t)$ and $\gamma_{2}(t)$ at the start of time slot $t$, which can be acquired by allocating two estimation periods; one for the BSU1 channel and the other for the BS-U2 channel, which is a huge improvement compared to the $K$ estimation periods that need to be allocated when the ICI needs to be estimated, see Sec. II-D.

\section{A. Proposed D-TFDD For Unknown ICI}

The throughput region of the BS-U1 and BS-U2 channels employing the D-TFDD scheme for unknown ICI is also given by (10) and 111, where $O_{1}^{m}(t)$ and $O_{2}^{l}(t)$ are defined in (8) and (9), respectively. The only difference now is that $q_{1}^{m}(t)$ and $q_{2}^{l}(t)$ in (10) and (11) are different when the D-TFDD for unknown ICI is applied. The optimal $q_{1}^{m}(t)$ and $q_{2}^{l}(t)$, which maximize the throughput region, defined by (10) and (11), of the D-TFDD scheme for unknown ICI are given in the following.

For the case when the ICI is unknown at the nodes, first we define

$$
\begin{aligned}
& m^{*} \triangleq \arg \max _{m}\left\{R_{1}^{m} O_{1, e}^{m}(t)\right\}, \\
& l^{*} \triangleq \arg \max _{l}\left\{R_{2}^{l} O_{2, e}^{l}(t)\right\},
\end{aligned}
$$

where $O_{1, e}^{m}(t)$ and $O_{2, e}^{l}(t)$ are defined as

$$
\begin{aligned}
& O_{1, e}^{m}(t)=\left\{\begin{array}{ll}
1 & \text { if } C_{1}^{e}(t) \geq R_{1}^{m} \\
0 & \text { if } C_{1}^{e}(t)<R_{1}^{m}
\end{array},\right. \\
& O_{2, e}^{l}(t)= \begin{cases}1 & \text { if } C_{2}^{e}(t) \geq R_{2}^{l} \\
0 & \text { if } C_{2}^{e}(t)<R_{2}^{l} .\end{cases}
\end{aligned}
$$

The variables, $C_{1}^{e}(t)$ and $C_{2}^{e}(t)$, used in (21) and 22), are defined as

$$
\begin{aligned}
& C_{1}^{e}(t)=\log _{2}\left(1+\frac{P_{1} \gamma_{1}(t)}{1+\gamma_{I 1}^{e}(t)}\right), \\
& C_{2}^{e}(t)=\log _{2}\left(1+\frac{P_{2} \gamma_{2}(t)}{1+\gamma_{I 2}^{e}(t)}\right),
\end{aligned}
$$

where $\gamma_{I 1}^{e}(t)$ and $\gamma_{I 2}^{e}(t)$ are given in Proposition 1 in the following, and they can be thought of as estimates of the ICI in time slot $t$. 
The optimal $q_{1}^{m}(t)$ and $q_{2}^{l}(t)$, which maximize the throughput region, defined by (10) and 111, of the D-TFDD for unknown ICI are as follows

BS-U1 transmission $\longrightarrow q_{1}^{m^{*}}(t)=1, q_{1}^{m}(t)=0, \forall m \neq m^{*}$ and $q_{2}^{l}(t)=0, \forall l$, if $\left[\Lambda_{1}(t) \geq \Lambda_{2}(t)\right.$ and $\left.\Lambda_{1}(t)>0\right]$,

BS-U2 transmission $\longrightarrow q_{2}^{l^{*}}(t)=1, q_{2}^{l}(t)=0, ; \forall l \neq l^{*}$, and $q_{1}^{m}(t)=0, \forall m$, if $\left[\Lambda_{2}(t) \geq \Lambda_{1}(t)\right.$ and $\left.\Lambda_{2}(t)>0\right]$,

Silence $q_{1}^{m}(t)=0, \forall m$ and $q_{2}^{l}(t)=0, \forall l$, if $\left[\Lambda_{1}(t)=0\right.$ and $\left.\Lambda_{2}(t)=0\right]$,

where $\Lambda_{1}(t)$ and $\Lambda_{2}(t)$ are given by

$$
\begin{aligned}
& \Lambda_{1}(t)=\mu C_{1}^{e}(t), \\
& \Lambda_{2}(t)=(1-\mu) C_{2}^{e}(t),
\end{aligned}
$$

and $m^{*}$ and $l^{*}$ are given by (19) and (20), respectively. In (26) and (27), $\mu$ is a priori given constant which satisfies $0 \leq \mu \leq$ 1. By varying $\mu$ from zero to one, the entire boundary line of the BS-U1 and BS-U2 throughput region can be obtained.

Proposition 1: The variables $\gamma_{I 1}^{e}(t)$ and $\gamma_{I 2}^{e}(t)$, found in the expressions in (23) and (24), which maximize the BS-U1 and BS-U2 throughput region of the D-TFDD scheme for unknown ICI proposed in (25) are found as follow

$$
\begin{aligned}
& \gamma_{I 1}^{e}(t+1)=\gamma_{I 1}^{e}(t)-\delta_{1}(t) \Phi_{1}(t), \\
& \gamma_{I 2}^{e}(t+1)=\gamma_{I 2}^{e}(t)-\delta_{2}(t) \Phi_{2}(t),
\end{aligned}
$$

where $\delta_{k}(t)$, for $k \in\{1,2\}$, can be some properly chosen monotonically decaying function of $t$ with $\delta_{k}(1)<1$, such as $\frac{1}{2 t}$. Furthermore, $\Phi_{1}(t)$ and $\Phi_{2}(t)$ in (28) and (29) are obtained as

$$
\begin{aligned}
& \Phi_{1}(t)=\frac{t-1}{t} \Phi_{1}(t-1) \\
& -\frac{1}{t} \frac{P_{1} \gamma_{1}(t) \delta_{1}^{m^{*}}(t) q_{1}^{m^{*}}(t)\left(2 \chi_{1}^{e}(t)\left[O_{1, e}^{m^{*}}(t)-O_{1}^{m^{*}}(t)\right]-\mu R_{1}^{m^{*}}\right)}{\ln (2)\left(1+\gamma_{I 1}^{e}(t)+P_{1} \gamma_{1}(t)\right)\left(1+\gamma_{I 1}^{e}(t)\right)}, \\
& \Phi_{2}(t)=\frac{t-1}{t} \Phi_{2}(t-1) \\
& -\frac{1}{t} \frac{P_{2} \gamma_{2}(t) \delta_{2}^{l^{*}}(t) q_{2}^{l^{*}}(t)\left(2 \chi_{2}^{e}(t)\left[O_{2, e}^{l^{*}}(t)-O_{2}^{l^{*}}(t)\right]-(1-\mu) R_{2}^{l^{*}}\right)}{\ln (2)\left(1+\gamma_{I 2}^{e}(t)+P_{2} \gamma_{2}(t)\right)\left(1+\gamma_{I 2}^{e}(t)\right)},
\end{aligned}
$$

respectively, where $\delta_{1}^{m^{*}}(t)$ and $\delta_{2}^{l^{*}}(t)$, are defined as

$$
\delta_{1}^{m^{*}}(t)=\left\{\begin{array}{l}
1 \text { if }\left(R_{1}^{m^{*}}-C_{1}^{e}(t-1)\right)\left(R_{1}^{m^{*}}-C_{1}^{e}(t)\right) \leq 0 \\
0 \text { if }\left(R_{1}^{m^{*}}-C_{1}^{e}(t-1)\right)\left(R_{1}^{m^{*}}-C_{1}^{e}(t)\right)>0,
\end{array}\right.
$$

and

$$
\delta_{2}^{l^{*}}(t)=\left\{\begin{array}{lll}
1 & \text { if }\left(R_{2}^{l^{*}}-C_{2}^{e}(t-1)\right) & \left(R_{2}^{l^{*}}-C_{2}^{e}(t)\right) \leq 0 \\
0 & \text { if }\left(R_{2}^{l^{*}}-C_{2}^{e}(t-1)\right) & \left(R_{2}^{l^{*}}-C_{2}^{e}(t)\right)>0
\end{array}\right.
$$

respectively. On the other hand, the variables $\chi_{1}^{e}(t)$ and $\chi_{2}^{e}(t)$ in (30) and (31) are calculated as

$$
\chi_{k}^{e}(t+1)=\chi_{k}^{e}(t)+\delta_{k}^{\chi}(t)\left[\bar{\epsilon}_{k}(t)-\epsilon\right]^{+}, k \in\{1,2\},
$$

where $\delta_{k}^{\chi}(t)$ for $k \in\{1,2\}$ can be some properly chosen monotonically decaying function of $t$ with $\delta_{k}^{\chi}(1)<1$, such as $\frac{1}{2 t}, \epsilon$ is a small constant which can be configured at the system level, and [.] ${ }^{+}$denotes only positive values. Moreover, in (34), $\bar{\epsilon}_{k}(t)$, for $k \in\{1,2\}$, are obtained as

$$
\begin{aligned}
& \bar{\epsilon}_{1}(t)=\frac{t-1}{t} \bar{\epsilon}_{U}(t-1)+\frac{1}{t} q_{1}^{m^{*}}(t)\left(O_{1}^{m^{*}}(t)-O_{1, e}^{m^{*}}(t)\right)^{2}, \\
& \bar{\epsilon}_{2}(t)=\frac{t-1}{t} \bar{\epsilon}_{D}(t-1)+\frac{1}{t} q_{2}^{l^{*}}(t)\left(O_{2}^{l^{*}}(t)-O_{2, e}^{l^{*}}(t)\right)^{2} .
\end{aligned}
$$

Finally, we note that, $\bar{\epsilon}_{k}(0), \Phi_{k}(0), \chi_{k}^{e}(0)$, and $\gamma_{I k}^{e}(0), k \in$ $\{1,2\}$, found in (28)-(36), are initialized to zero. The variables $\Phi_{1}(t), \Phi_{2}(t), \chi_{1}^{e}(t+1), \chi_{2}^{e}(t+1), \delta_{1}^{m^{*}}(t), \delta_{2}^{l^{*}}(t), \bar{\epsilon}_{1}(t)$ and $\bar{\epsilon}_{2}(t)$ are auxiliary variables used for real-time estimation of the ICIs $\gamma_{I 1}^{e}(t)$ and $\gamma_{I 2}^{e}(t)$.

The D-TFDD scheme for unknown ICI is provided in an algorithmic form in Algorithm 1

Algorithm 1 Finding the optimal decision variables, $q_{1}^{m}, \forall m$, and $q_{2}^{l}(t), \forall l$, for a given value of $\mu$.

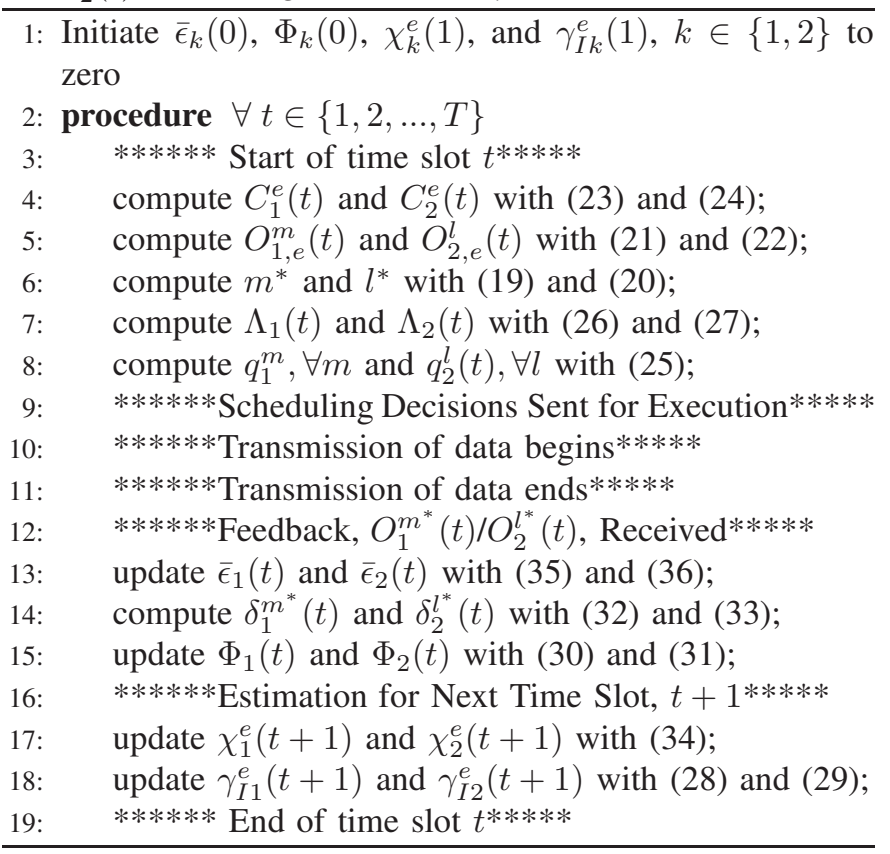

Proof: Please refer to Appendix $\mathrm{C}$ for the proof.

Remark 3: All equations from (19) to (36) are straightforward calculations that do not depend on any hidden function or loop routines. As a result, the complexity of Algorithm 1 grows linearly with $M$ or $L$.

Remark 4: Note that for the D-TFDD scheme for unknown ICI proposed above, the functions $\Phi_{1}(t)$ and $\Phi_{2}(t)$ need to be calculated at the end of time slot $t$. To this end, note that all the variables in (30) and (31) are known at the end of time time slot $t$. In particular, the outage variables $O_{1}^{m^{*}}(t)$ and $O_{2}^{l^{*}}(t)$ either are already available at the BS if the BS is the receiver node at the end of time slot $t$, or are computed at the BS by a 1-bit feedback from the transmitting node. Moreover, $O_{1}^{m^{*}}(t)=1$ if the message received by the receiving node of the BS-U1 channel is decoded successfully in time slot $t$, otherwise $O_{1}^{m^{*}}(t)=0$. Similarly, $O_{2}^{l^{*}}(t)=1$ if the 
message received by the receiving node of the BS-U2 channel is decoded successfully in time slot $t$, otherwise $O_{2}^{l^{*}}(t)=0$. Hence, the BS is able to calculate (30) and 31) at the end of time slot $t$, compute $\gamma_{I 1}^{e}(t+1)$ and $\gamma_{I 2}^{e}(t+1)$ by using (28) and 29) at the end of time slot t, and use it at the start of time slot $t+1$ by plugging in 23) and 24, then plug 23 and (24) into 26) and (27), respectively, and thereby be able to compute the selection variables in 25).

Remark 5: If the statistical characteristics of the ICI remain constant over time, $\gamma_{I 1}^{e}(t)$ and $\gamma_{I 2}^{e}(t)$ in (28) and (29) converge to constants such as $\lim _{t \rightarrow \infty} \gamma_{I 1}^{e}(t)=\gamma_{I 1}$ and $\lim _{t \rightarrow \infty} \gamma_{I 2}^{e}(t)=\gamma_{I 2}$. On the other hand, if the statistical characteristics of the ICI is slowly changing over time, the proposed D-TFDD still works.

\section{B. Diversity Gain of the Proposed D-TFDD for Unknown ICI}

It is quite interesting to investigate the diversity gain that the D-TFDD scheme for unknown ICI, proposed in Section IV-A achieves. In the following, we derive the asymptotic outage probabilities of the BS-U1 and the BS-U2 channels, achieved with the D-TFDD scheme for unknown ICI proposed in Sec. IV-A for the case when $\mu=\frac{1}{2}, M=L=1$, and $R_{1}^{1}=R_{2}^{1}=R_{0}$.

Theorem 3: The asymptotic outage probability of the $\mathrm{D}$ TFDD scheme for unknown ICI, proposed in Section IV-A for the case of Rayleigh fading and when $\mu=\frac{1}{2}, M=L=1$, $P_{1}=P_{2}=P$, and $R_{1}^{1}=R_{2}^{1}=R_{0}$ hold, is given by

$$
\begin{gathered}
P_{\text {out }} \rightarrow \frac{\gamma_{\text {th }}^{2}}{\Omega_{0}^{2}}\left[\hat{\Omega}_{I 1}+\hat{\Omega}_{I 2}+\hat{\Omega}_{I S}\right], \text { as } P \rightarrow \infty, \\
\text { where } \gamma_{\text {th }}=\frac{2^{R_{0}}-1}{P}, \Omega_{0}=E\left\{\frac{\left|h_{1}(t)\right|^{2}}{\sigma_{1}^{2}\left(1+\gamma_{I 1}\right)}\right\}=E\left\{\frac{\left|h_{2}(t)\right|^{2}}{\sigma_{2}^{2}\left(1+\gamma_{I 2}\right)}\right\}, \\
\hat{\Omega}_{I 1}=\Omega_{I}\left(\frac{1+\gamma_{I 2}}{1+\gamma_{I 1}}\right)\left(\sum_{n=0}^{K-1}\left[1+(n+1) \Omega_{I}\right] e^{-\frac{\gamma_{I 1}}{\Omega_{I}}} \sum_{t=0}^{n} \frac{\left(\frac{\gamma_{I 1}}{\Omega_{I}}\right)^{i}}{t !}\right),
\end{gathered}
$$

$\hat{\Omega}_{I 2}=\Omega_{I}\left(\frac{1+\gamma_{I 1}}{1+\gamma_{I 2}}\right)\left(\sum_{n=0}^{K-1}\left[1+(n+1) \Omega_{I}\right] e^{-\frac{\gamma_{I 2}}{\Omega_{I}}} \sum_{t=0}^{n} \frac{\left(\frac{\gamma_{I 2}}{\Omega_{I}}\right)^{i}}{t !}\right)$,

$\hat{\Omega}_{I S}=\left(1+\gamma_{I 1}\right)\left(1+\gamma_{I 2}\right)$,

$\Omega_{I}=E\left\{\gamma_{I 1}(t)\right\}=E\left\{\gamma_{I 2}(t)\right\}$.

In the above expression, $\gamma_{I 1}$ and $\gamma_{I 2}$ are constant values obtained as $\lim \gamma_{I 1}^{e}(t)=\gamma_{I 1}$ and $\lim _{t \rightarrow \infty} \gamma_{I 2}^{e}(t)=\gamma_{I 2}$. It is clear from $\sqrt{37 \overrightarrow{7}}$ that $P_{\text {out }}$ has a diversity gain of two.

Proof: Please refer to Appendix $\mathrm{D}$ for the proof.

The result in Theorem 3 shows that the D-TFDD scheme for unknown ICI proposed in Sec. IV-A achieves double the diversity gain compared to existing duplexing schemes, which leads to very large performance gains. Moreover, Theorem 3 shows that doubling of the diversity gain on both the BS$\mathrm{U} 1$ and BS-U2 channels is achievable even when there is no ICI knowledge at the nodes, which is a very interesting result that shows the superior performance of the D-TFDD scheme for unknown ICI proposed in Sec. III-A compared to existing duplexing schemes.

\section{Simulation And Numerical Results}

In this section, we evaluate the performance of the proposed D-TFDD scheme for unknown ICI and its upper bound, the proposed D-TFDD scheme for known ICI. Then, we compare its performance to the performance achieved with a static-TDD scheme and to the state-of-the-art D-TDD and DFDD schemes. To this end, we first introduce the benchmark schemes and then present the numerical results.

\section{A. Benchmark Schemes}

1) Static-TDD: In the static-TDD scheme, see [2], the BS receives and transmits in prefixed time slots. Assuming single transmission rates at the transmitting nodes of the BS-U1 and BS-U2 channels, and assuming that that the fractions of the total number of time slots, $T$, allocated on the BS-U1 and BS-U2 channels are $\mu$ and $1-\mu$, respectively, e.g., channel BS-U1 is active in the first $\mu T$ and channel BS-U2 is active in following $(1-\mu) T$ time slots, the BS-U1 and BS-U2 throughput during $T \rightarrow \infty$ time slots, is given by

$$
\bar{R}_{k}=\lim _{T \rightarrow \infty} \frac{\mu_{k}}{T} \sum_{t=1}^{T} O_{k}^{1}(t) R_{k}^{1}, k \in\{1,2\},
$$

and the outage probability is given by

$$
P_{\text {out }}=1-\lim _{T \rightarrow \infty}\left(\frac{1}{T} \sum_{t=1}^{\mu T} O_{1}^{1}(t)+\sum_{t=\mu T+1}^{T} O_{2}^{1}(t)\right),
$$

where $O_{k}^{1}(t), k \in\{1,2\}$, is defined as

$$
O_{k}^{1}(t)=\left\{\begin{array}{cc}
1 & \text { if } \log _{2}\left(1+\frac{P_{k}}{\mu_{k}} \gamma_{k}(t)\right) \geq R_{k}^{1} \\
0 & \text { if } \log _{2}\left(1+\frac{P_{k}}{\mu_{k}} \gamma_{k}(t)\right)<R_{k}^{1} .
\end{array}\right.
$$

2) D-TDD Scheme: The distributed D-TDD scheme proposed in [25] is considered as a benchmark for comparison. We note that this scheme requires full knowledge of the ICI, which, as argued in Section II-D is not practical. In addition, we note that this scheme without ICI knowledge transforms to the static-TDD. Hence, the practical distributed TDD scheme is the static-TDD since it does not need ICI knowledge. The distributed D-TDD scheme in [25] can serve only as an upper bound to the practical static-TDD.

Note that D-TDD and D-FDD schemes only different in how they share the time and frequency resources, but achieve the same performance. Therefore, for comparison purposes, we can use either of them. In the following, we choose the D-TDD scheme.

\section{B. Numerical Results}

All of the results presented in this section have been performed by numerical evaluation of the derived results and are confirmed by Monte Carlo simulations. Moreover, Rayleigh fading for the BS-U1 and BS-U2 channels, and Chi-square distribution for the ICI at the receiving nodes of the BS-U1 and BS-U2 channels are assumed. In all the numerical examples in Figs. 5],7, we assume $M=L$ and $R_{1}^{k}=R_{2}^{k}=k R$, for 


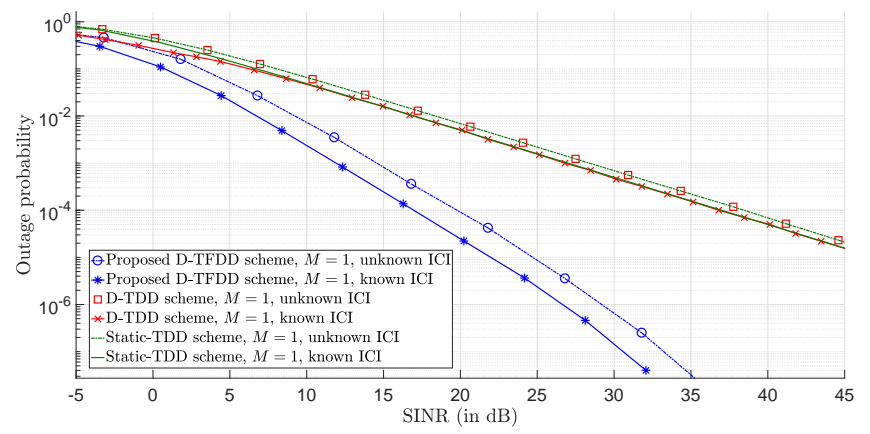

Fig. 5. Outage probability of the proposed D-TFDD schemes. Local-CSI and Full-CSI labels highlight that the corresponding schemes are without and with ICI knowledge, respectively.

$k=1,2, \ldots, M$, where $R$ is defined differently depending on the corresponding example. The signal to interference plus noise ratio (SINR) is defined as the ratio of the average received signal power to interference power plus the noise power.

1) Constraint on the Average Transmit Power: In the numerical examples, we select the fixed powers $P_{1}$ and $P_{2}$ such that the following long-term power constraints hold

$$
\begin{aligned}
\lim _{T \rightarrow \infty} \frac{1}{T} \sum_{t=1}^{T} \sum_{m=1}^{M} q_{1}^{m}(t) P_{1} \leq \bar{P}_{1} \\
\text { and } \lim _{T \rightarrow \infty} \frac{1}{T} \sum_{t=1}^{T} \sum_{l=1}^{L} q_{2}^{l}(t) P_{2} \leq \bar{P}_{2} .
\end{aligned}
$$

This enables all duplexing schemes to use identical average transmit powers, and thereby enable fair comparison between the different schemes.

2) Outage Probability: In Fig. 5, we illustrate the outage probabilities of the proposed D-TFDD schemes for unknown ICI and its upper bound, the D-TFDD for known ICI, as well as the benchmark schemes as a function of the SINR for $M=$ $1, \mu=\frac{1}{2}$, where $R$ is set to $R=1 \mathrm{bits} / \mathrm{symb}$. As predicted by Theorems 2 and 3 Fig. 5 shows that the proposed D-TFDD schemes for unknown and known ICI achieve double the diversity gain compared to the benchmark schemes. Intuitively, the doubling of the diversity gain occurs since the proposed D-TFDD schemes can select for transmission between two independent channels, in each time slot, compared to the existing D-TDD and D-FDD system, which can select between two dependent channels, in each time slot. The doubling of the diversity gain leads to very large performance gains in terms of SINR. For example, SINR gains of $10 \mathrm{~dB}$ and 15 $\mathrm{dB}$ can be achieved for outage probabilities of $10^{-2}$ and $10^{-3}$, respectively. On the other hand, the proposed D-TFDD schemes for unknown ICI has around $3 \mathrm{~dB}$ penalty loss compared its upper bound, the proposed D-TFDD schemes for known ICI. This example shows the large performance gains of the proposed D-TFDD scheme with unknown ICI compared to existing D-TDD and/or static-TDD schemes.

3) Throughput Region: For the example in Fig. 6users have ominidirectional antenna with unity gain and the BS has a directional antenna with gain of $16 \mathrm{dBi}$. The power at the users is set to $24 \mathrm{dBm}$ and the power at BS is set to $46 \mathrm{dBm}$, and we use the proposed scheme with Type 1 and Type 2 for this example. The distances between U1 and BS, as well as BS and $\mathrm{U} 2$, are assumed to be fixed and set to $700 \mathrm{~m}$. The noise figure of $\mathrm{BS}$ and $\mathrm{U} 2$ are set to $2 \mathrm{~dB}$ and $7 \mathrm{~dB}$, respectively. The above parameters reflect the parameters used in practice. In addition, the mean power of the channel gains of the BS-U1 and BS-U2 channels are calculated using the standard pathloss model as [29]-[31]

$$
E\left\{\left|h_{k}(t)\right|^{2}\right\}=\left(\frac{c}{4 \pi f_{c}}\right)^{2} d_{k}^{-\beta}, \text { for } k \in\{1,2\},
$$

where $c$ is the speed of light, $f_{c}$ is the carrier frequency, $d_{k}$ is the distance between the transmitter and the receiver of link $k$, and $\beta$ is the path loss exponent. Moreover, the carrier frequency is set to $f_{c}=1.9 \mathrm{GHz}$, and we assume $\beta=3.6$ for the BS-U1 and BS-U2 channels.

In Fig. 6, we show the throughput region achieved with the proposed D-TFDD schemes for unknown and known ICI with $M=1$, for two different scenarios, one with high interference, $\mathrm{SINR}=10 \mathrm{~dB}$, and the other one with low interference, SINR=20 dB. Furthermore, we show the throughput regions achieved with the benchmark schemes. For the proposed and the benchmark schemes the value of $R$ is optimized numerically for a given $\mu$ such that the throughput is maximized. As can be seen from Fig. 6, the proposed D-TDD scheme without ICI knowledge achieves almost the exact throughput region as its upper bound achieved with DTFDD with full ICI knowledge for $\mathrm{SINR}=20 \mathrm{~dB}$. Also, in the relatively high interference region, i.e., $S I N R=10 \mathrm{~dB}$, the proposed D-TFDD scheme without ICI knowledge achieves a throughput region which is very close to its upper bound achieved with the D-TFDD scheme with ICI knowledge. On the other hand, the throughput that the proposed D-TFDD scheme for unknown ICI achieves is close or higher than the throughput achieved with the benchmark D-TDD scheme with ICI knowledge, which is an interesting result since the proposed scheme without ICI knowledge wastes only two time slots compared to the $K$ time slots that the D-TDD and DFDD schemes with ICI knowledge waste. More importantly, the gains that the proposed D-TFDD scheme for unknown ICI achieves compared to the benchmark schemes without ICI knowledge are considerable. For example, the proposed D-TFDD scheme for unknown ICI has a BS-U1 throughput gain of about $66 \%, 80 \%$ and $100 \%$, for $\mathrm{SINR}=10 \mathrm{~dB}$, and about $17 \%, 18 \%$ and $38 \%$, for SINR=20 dB, compared to the existing D-TDD and to the static-TDD schemes without ICI knowledge for a BS-U2 throughput of 2, 3, and 4 bits/symb, respectively.

4) Sum Throughput: In Fig. 7 we illustrate the sum of the BS-U1 and BS-U2 throughputs achieved with the proposed D-TFDD scheme for unknown ICI and the static-TDD as a function of the SINR for $M=1,4,16, \infty$, where $R$ is set to $R=10 / M$ bits/symb. From Fig. 7 we can see that by increasing $M$ from 1 to 4 in the proposed D-TFDD scheme for unknown ICI, we can gain more than $10 \mathrm{dBs}$ in SINR for around 4 bits/symb sum throughput. Whereas, by increasing $M$ from 4 to 16 we can gain an additional $1 \mathrm{~dB}$ in SINR for around 4 bits/symb of the sum throughput. Moreover, 

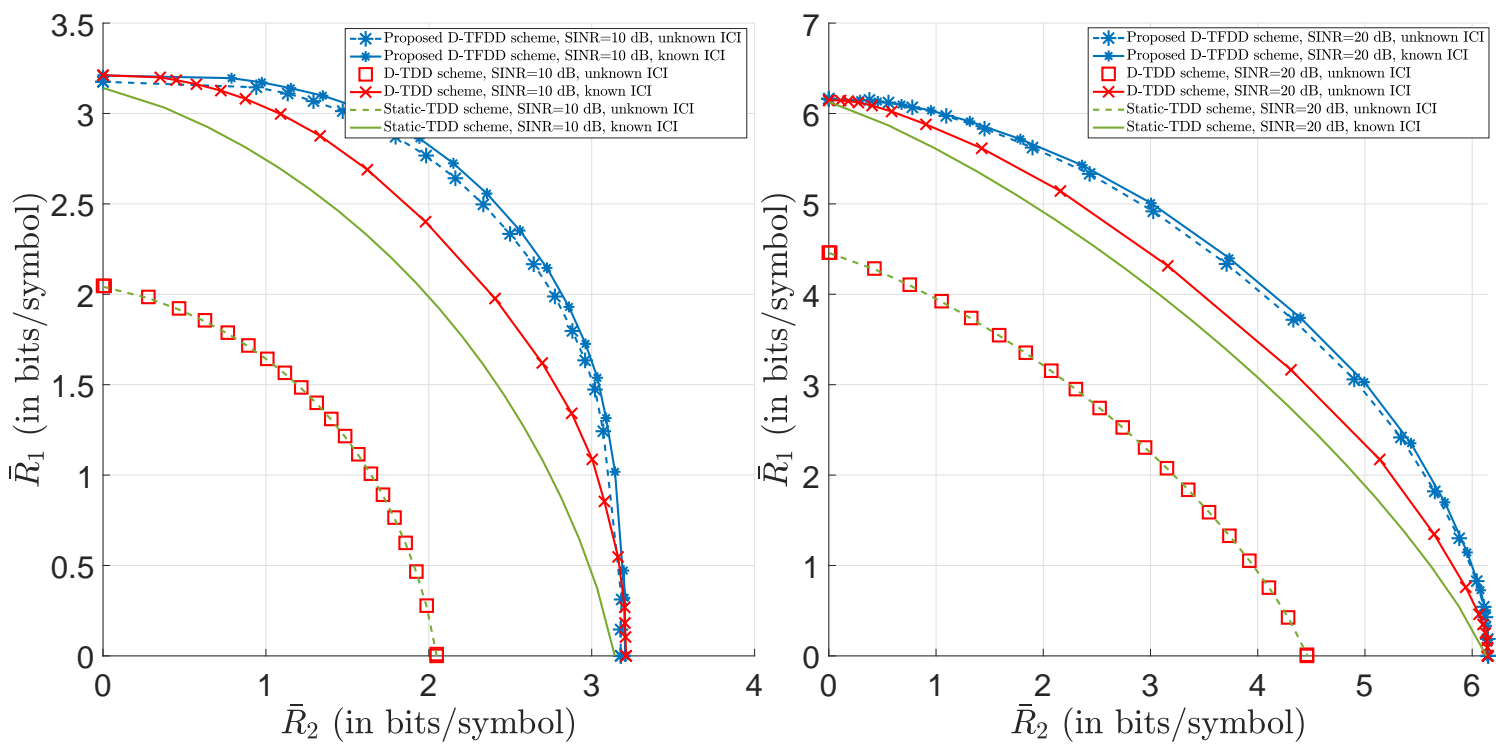

Fig. 6. Throughput regions of the proposed D-TFDD schemes: left for SINR=10 dB, and right for SINR=20 dB.

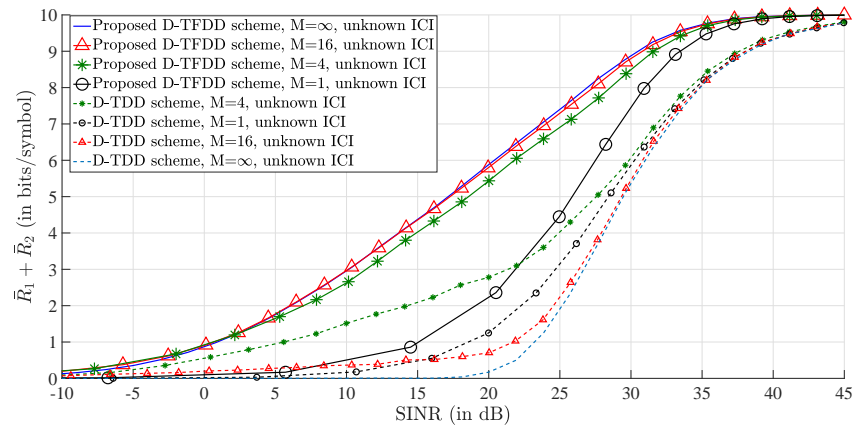

Fig. 7. Throughputs vs. SINR of the proposed D-TFDD schemes with and without ICI knowledge with different discrete-rates quantization level.

the proposed D-TFDD scheme for unknown ICI achieves substantial gains compared to the static-TDD. For example, about $3 \mathrm{~dB}$ and $10 \mathrm{~dB}$ SINR gain is achieved for $M=1$ and $M=4$, respectively, for around 4 bits/symb sum throughput. Finally, Fig. 7 shows that the proposed scheme for $M=4$ performs very close to the case when $M=\infty$. Note that in Fig. 7) the throughputs saturate since as a function of $M$, the transmission rates are $10 / M$ bits/symb. Hence, the maximum possible transmission rate is $10 \mathrm{bits} / \mathrm{symb}$, even if the channels are error-free, which is the case in the high SINR.

The above numerical examples show that the proposed DTFDD scheme provides double the diversity gain compared to existing TDD/FDD schemes, which improves that reliability of the communication. Moreover, since the proposed D-TFDD scheme works in a distributed fashion, it does not need any coordination of the BSs. Another strength is that is does not require ICI estimation, which makes it practical for implementation. Finally, the proposed D-TFDD scheme fits well into the scope of $5 \mathrm{G}$. On the other hand, a weakness of the proposed D-TFDD scheme is the requirement of local CSI of the U1BS and U2-BS channels at the BS, which entails signalling overhead.

\section{CONCLUSION}

In this paper, we proposed a distributed D-TFDD scheme for unknown ICI. Using the proposed D-TFDD scheme, in a given frequency band, the BS adaptively selects to either communicate with $\mathrm{U} 1$ or with $\mathrm{U} 2$ in a given time slot based on the qualities of the BS-U1 and BS-U2 channels without ICI knowledge such that the BS-U1 and BS-U2 throughput region is maximized. We have shown that the proposed DTFDD scheme provides significant throughput and outage probability gains compared to the conventional static-TDD scheme, as well as to the D-TDD and D-FDD schemes. Moreover, we observed the the proposed D-TFDD scheme doubles the diversity gain on both the BS-U1 and BS-U2 channels compared to existing duplexing schemes, even when the ICI is unknown, which leads to very large performance gains.

\section{APPENDIX}

\section{A. Proof of Theorem 1}

Constraints $\mathrm{C} 1, \mathrm{C} 2$ and $\mathrm{C} 3$ in (12) make the problem nonconvex. To solve (12), we first we relax these constraints to $0 \leq q_{1}^{m}(t) \leq 1,0 \leq q_{2}^{l}(t) \leq 1$, and $0 \leq \sum_{m=1}^{M} q_{1}^{m}(t)+$ $\sum_{l=1}^{L} q_{2}^{l}(t) \leq 1$, thereby making the relaxed problem convex. The solutions of the relaxed convex problem is then shown to be such that $q_{1}^{m}(t)$ and $q_{2}^{l}(t)$ take the limiting values 0 or 1 , and not the values between 0 and 1 . As a result, the relaxed convex problem is equivalent to the original problem. To solve the relaxed problem, we use the Lagrangian. Thereby, 
we obtain

$$
\begin{aligned}
\mathcal{L}= & -\lim _{T \rightarrow \infty} \frac{1}{T} \sum_{t=1}^{T} \sum_{m=1}^{M} \mu R_{1}^{m} q_{1}^{m}(t) O_{1}^{m}(t) \\
& -\lim _{T \rightarrow \infty} \frac{1}{T} \sum_{t=1}^{T} \sum_{l=1}^{L}(1-\mu) R_{2}^{l} q_{2}^{l}(t) O_{2}^{l} \\
& -\sum_{m=1}^{M} \lambda_{1}^{m}(t) q_{1}^{m}(t)-\left(1-\sum_{m=1}^{M} \lambda_{2}^{m}(t) q_{1}^{m}(t)\right) \\
& -\sum_{l=1}^{L} \lambda_{3}^{l}(t) q_{2}^{l}(t)-\left(1-\sum_{l=1}^{L} \lambda_{4}^{l}(t) q_{2}^{l}(t)\right) \\
& -\lambda_{5}(t)\left(\sum_{m=1}^{M} q_{1}^{m}(t)+\sum_{l=1}^{L} q_{2}^{l}(t)\right) \\
& -\lambda_{6}(t)\left(1-\sum_{m=1}^{M} q_{1}^{m}(t)-\sum_{l=1}^{L} q_{2}^{l}(t)\right)
\end{aligned}
$$

where $\lambda_{1}^{m}(t) \geq 0, \lambda_{2}^{m}(t) \geq 0, \lambda_{3}^{l}(t) \geq 0, \lambda_{4}^{l}(t) \geq 0, \lambda_{5}(t) \geq$ 0 , and $\lambda_{6}(t) \geq 0, \forall m, l, i$, are the Lagrangian multipliers. Next, we rewrite (47) as

$$
\begin{aligned}
\mathcal{L}= & -\lim _{T \rightarrow \infty} \frac{1}{T} \sum_{t=1}^{T} \mu q_{1}(t) \max _{m}\left\{R_{1}^{m} O_{1}^{m}(t)\right\} \\
& -\lim _{T \rightarrow \infty} \frac{1}{T} \sum_{t=1}^{T}(1-\mu) q_{2}(t) \max _{l}\left\{R_{2}^{l} O_{2}^{l}(t)\right\} \\
& -\sum_{m=1}^{M} \lambda_{1}^{m}(t) q_{1}^{m}(t)-\left(1-\sum_{m=1}^{M} \lambda_{2}^{m}(t) q_{1}^{m}(t)\right) \\
& -\sum_{l=1}^{L} \lambda_{3}^{l}(t) q_{2}^{l}(t)-\left(1-\sum_{l=1}^{L} \lambda_{4}^{l}(t) q_{2}^{l}(t)\right) \\
& -\lambda_{5}(t)\left(q_{1}(t)+q_{2}(t)\right)-\lambda_{6}(t)\left(1-q_{1}(t)-q_{2}(t)\right) .
\end{aligned}
$$

Now, using (48) and defining $-\lambda_{5}(t)+\lambda_{6}(t) \triangleq \beta(t)$, we can find the optimal state-selection variables $q_{1}^{m}(t)$ and $q_{2}^{l}(t)$ as follows. The conditions which maximize (47), in the cases when the transmit node on the BS-U1 channel transmits with $R_{1}^{m}$ and the transmit node on the BS-U2 channel is silent, are

$$
\begin{gathered}
{\left[\mu \max _{m}\left\{R_{1}^{m} O_{1}^{m}(t)\right\}-\beta(t)>0\right]} \\
\text { and }\left[(1-\mu) \max _{l}\left\{R_{2}^{l} O_{2}^{l}(t)\right\}-\beta(t)<0\right] .
\end{gathered}
$$

On the other hand, the conditions for maximizing (48) in the case when the transmit node on the BS-U2 channel transmits with $R_{2}^{l}$ and the transmit node on the BS-U1 channel is silent, are

$$
\begin{gathered}
{\left[\mu \max _{m}\left\{R_{1}^{m} O_{1}^{m}(t)\right\}-\beta(t)<0\right]} \\
\text { and }\left[(1-\mu) \max _{l}\left\{R_{2}^{l} O_{2}^{l}(t)\right\}-\beta(t)>0\right] .
\end{gathered}
$$

In (49) and (50), we can substitute $\mu R_{1}^{m} O_{1}^{m}(t)$ with $\Lambda_{1}^{m}(t)$ and $(1-\mu) R_{2}^{l} O_{2}^{l}(t)$ with $\Lambda_{2}^{l}(t)$, and thereby obtain $q_{1}^{m}(t)$ and $q_{2}^{l}(t)$ as in 13). This completes the proof.

\section{B. Proof of Theorem 2}

In time slot $t$, an outage occurs if the BS-U1 channel is selected for transmission and the BS-U1 channel is too weak to support the rate $R_{0}$, i.e., $q_{1}^{1}(t)=1$ and $O_{1}^{1}(t)=0$, or if the BS-U2 channel is selected to transmit and the BS-U2 channel is too weak to support the rate $R_{0}$, i.e., $q_{2}^{1}(t)=1$ and $O_{2}^{1}(t)=0$, or if both the BS-U1 the BS-U2 channels are not selected for transmission in time slot $t$, i.e., if $q_{1}^{1}(t)=$ $q_{2}^{1}(t)=0$, since in that case the time slot $t$ is wasted. Hence, the outage probability $P_{\text {out }}$ can be obtained as

$$
\begin{gathered}
P_{\text {out }}=\operatorname{Pr}\left\{\left[q_{1}^{1}(t)=1 \text { AND } O_{1}^{1}(t)=0\right]\right. \\
\text { OR }\left[q_{2}^{1}(t)=1 \text { AND } O_{2}^{1}(t)=0\right] \\
\text { OR } \left.\left[q_{1}^{1}(t)=q_{2}^{1}(t)=0\right]\right\} \\
\stackrel{(a)}{=} \operatorname{Pr}\left\{q_{1}^{1}(t)=1 \text { AND } O_{1}^{1}(t)=0\right\} \\
+\operatorname{Pr}\left\{q_{2}^{1}(t)=1 \text { AND } O_{2}^{1}(t)=0\right\} \\
+\operatorname{Pr}\left\{q_{1}^{1}(t)=q_{2}^{1}(t)=0\right\},
\end{gathered}
$$

where $(a)$ follows since the events $q_{1}^{1}(t)=1$ and $q_{1}^{1}(t)=0$, and also the events $q_{2}^{1}(t)=1$ and $q_{2}^{1}(t)=0$ are mutually exclusive. Since $\mu=\frac{1}{2}, \Lambda_{1}^{1}(t)$ and $\Lambda_{2}^{1}(t)$ in (14) and (15) simplify to

$$
\Lambda_{k}^{1}(t)=\frac{1}{2} R_{0} O_{k}^{1}(t), k \in\{1,2\} .
$$

Now, inserting $\Lambda_{1}^{1}(t)$ and $\Lambda_{2}^{1}(t)$ from (52) into (13), we obtain that $q_{1}^{1}(t)=1$ if $O_{1}^{1}(t) \geq O_{2}^{1}(t)$ and $O_{1}^{1}(t)>0$, which means that $q_{1}^{1}(t)=1$ occurs if $O_{1}^{1}(t)=1$. Hence, the event $q_{1}^{1}(t)=1$ and $O_{1}^{1}(t)=0$ is an impossible event, thereby leading to $\operatorname{Pr}\left\{q_{2}^{1}(t)=1\right.$ AND $\left.O_{2}^{1}(t)=0\right\}=0$ in (51). Similarly, we can conclude that $\operatorname{Pr}\left\{q_{1}^{1}(t)=1\right.$ AND $\left.O_{1}^{1}(t)=0\right\}=0$ in (51). Next, we obtain that $q_{1}^{1}(t)=q_{2}^{1}(t)=0$ occurs iff $O_{1}^{1}(t)=$ $O_{2}^{1}(t)=0$ holds, thereby leading to $\operatorname{Pr}\left\{q_{1}^{1}(t)=q_{2}^{1}(t)=0\right\}=$ $\operatorname{Pr}\left\{O_{1}^{1}(t)=0\right.$ AND $\left.O_{2}^{1}(t)=0\right\}$ in [51). Inserting this into (51), we obtain

$$
\begin{aligned}
P_{\text {out }} & =\operatorname{Pr}\left\{O_{1}^{1}(t)=0 \text { AND } O_{2}^{1}(t)=0\right\} \\
& =\operatorname{Pr}\left\{\log _{2}\left(1+P \frac{\left|\gamma_{1}(t)\right|^{2}}{1+\gamma_{I 1}(t)}\right)<R_{0}\right. \\
& \text { AND } \left.\log _{2}\left(1+P \frac{\left|\gamma_{2}(t)\right|^{2}}{1+\gamma_{I 2}(t)}\right)<R_{0}\right\} \\
& =\operatorname{Pr}\left\{\frac{\left|\gamma_{1}(t)\right|^{2}}{1+\gamma_{I 1}(t)}<\gamma_{\text {th }} \text { AND } \frac{\left|\gamma_{2}(t)\right|^{2}}{1+\gamma_{I 2}(t)}<\gamma_{\text {th }}\right\},
\end{aligned}
$$

where $\gamma_{\mathrm{th}}=\frac{2^{R_{0}}-1}{P}$. The variables $\gamma_{1}(t)$ and $\gamma_{2}(t)$ have identical and independent exponential distributions with PDFs denoted by $f_{\gamma_{1}}\left(\gamma_{1}\right)$ and $f_{\gamma_{2}}\left(\gamma_{2}\right)$, respectively, both with mean $\Omega_{0}=E\left\{\frac{\left|h_{1}(t)\right|^{2}}{\sigma_{1}^{2}}\right\}=E\left\{\frac{\left|h_{2}(t)\right|^{2}}{\sigma_{2}^{2}}\right\}$. On the other hand, the variables $\gamma_{I 1}(t)$ and $\gamma_{I 2}(t)$ have identical yet dependent exponential distributions with joint PDF denoted by $f_{\gamma_{I 1}, \gamma_{I 2}}\left(Z_{1}, Z_{2}\right)$. As a result, (53) can be obtained as

$$
\begin{aligned}
P_{\text {out }}= & \int_{0}^{\infty} \int_{0}^{\infty} \int_{0}^{\gamma_{\text {th }}\left(1+Z_{1}\right)} \int_{0}^{\gamma_{\text {th }}\left(1+Z_{2}\right)} f_{\gamma_{1}, \gamma_{2}, \gamma_{I 1}, \gamma_{I 2}}\left(\gamma_{1}, \gamma_{2}, Z_{1}, Z_{2}\right) \\
& \times d \gamma_{1} d \gamma_{2} d Z_{1} d Z_{2} .
\end{aligned}
$$

We can rewrite $f_{\gamma_{1}, \gamma_{2}, \gamma_{I 1}, \gamma_{I 2}}\left(\gamma_{1}, \gamma_{2}, Z_{1}, Z_{2}\right)$ in (54) as

$$
\begin{aligned}
f_{\gamma_{1}, \gamma_{2}, \gamma_{I 1}, \gamma_{I 2}} & \left(\gamma_{1}, \gamma_{2}, Z_{1}, Z_{2}\right)=f_{\gamma_{1} \mid \gamma_{I 1}, \gamma_{I 2}}\left(\gamma_{1}\right) \\
& \times f_{\gamma_{2} \mid \gamma_{I 1}, \gamma_{I 2}}\left(\gamma_{2}\right) f_{\gamma_{I 1}, \gamma_{I 2}}\left(Z_{1}, Z_{2}\right),
\end{aligned}
$$

since $\gamma_{1}$ and $\gamma_{2}$ have independent distributions. By substituting the PDFs of $\gamma_{1}$ and $\gamma_{2}$ into (55), then inserting (55) into (54), 
we can obtain (54) as

$$
\begin{aligned}
P_{\text {out }}= & \int_{0}^{\infty} \int_{0}^{\infty} \frac{1}{\Omega_{0}} \int_{0}^{\gamma_{\mathrm{th}}\left(1+Z_{2}\right)} e^{-\left(\frac{\gamma_{2}}{\Omega_{0}}\right)} d \gamma_{2} \times \frac{1}{\Omega_{0}} \int_{0}^{\gamma_{\mathrm{th}}\left(1+Z_{1}\right)} e^{-\left(\frac{\gamma_{1}}{\Omega_{0}}\right)} d \gamma_{1} \\
& \times f_{\gamma_{I 1}, \gamma_{I 2}}\left(Z_{1}, Z_{2}\right) d Z_{1} d Z_{2} .
\end{aligned}
$$

In (56), by integrating over $\gamma_{1}$ and $\gamma_{2}$, and letting $P \rightarrow \infty$ (consequently $\gamma_{\text {th }} \rightarrow 0$ ), we obtain

$$
\begin{aligned}
P_{\text {out }} \rightarrow \int_{0}^{\infty} \int_{0}^{\infty} \frac{\gamma_{\mathrm{th}}\left(1+Z_{2}\right)}{\Omega_{0}} \times \frac{\gamma_{\mathrm{th}}\left(1+Z_{1}\right)}{\Omega_{0}} \\
\quad \times f_{\gamma_{I 1}, \gamma_{I 2}}\left(Z_{1}, Z_{2}\right) d Z_{1} d Z_{2} \text { as } P \rightarrow \infty .
\end{aligned}
$$

Finally, by replacing

$$
\begin{aligned}
& E\left\{\left(1+\gamma_{I 1}(t)\right)\left(1+\gamma_{I 2}(t)\right)\right\} \\
& =\int_{0}^{\infty} \int_{0}^{\infty}\left(1+Z_{2}\right) \times\left(1+Z_{1}\right) \times f_{\gamma_{I 1}, \gamma_{I 2}}\left(Z_{1}, Z_{2}\right) d Z_{1} d Z_{2}
\end{aligned}
$$

into (57), the outage is obtained as in (18). This completes the proof.

\section{Proof of Proposition 1$]$}

The BS-U1 and BS-U2 throughputs are given in 10 and (11), respectively. However, since we can not compute $O_{1}^{m}(t)$ and $O_{2}^{l}(t)$, instead of (10) and (11), we use estimates for (10) and 11, given by

$$
\begin{aligned}
\bar{R}_{1, e} & =\lim _{T \rightarrow \infty} \frac{1}{T} \sum_{t=1}^{T} \sum_{m=1}^{M} R_{1}^{m} q_{1}^{m}(t) O_{1, e}^{m}(t), \\
\bar{R}_{2, e} & =\lim _{T \rightarrow \infty} \frac{1}{T} \sum_{t=1}^{T} \sum_{l=1}^{L} R_{2}^{l} q_{2}^{l}(t) O_{2, e}^{l}(t) .
\end{aligned}
$$

The accuracy of the estimates $\bar{R}_{1, e}$ and $\bar{R}_{2, e}$, depends on the following expressions

$$
\begin{aligned}
& \delta_{1}=\lim _{T \rightarrow \infty} \frac{1}{T} \sum_{t=1}^{T} q_{1}^{m}(t)\left(O_{1}^{m}(t)-O_{1, e}^{m}(t)\right)^{2} . \\
& \delta_{2}=\lim _{T \rightarrow \infty} \frac{1}{T} \sum_{t=1}^{T} q_{2}^{l}(t)\left(O_{2}^{l}(t)-O_{2, e}^{l}(t)\right)^{2} .
\end{aligned}
$$

Hence, 61 and 62 express the average of the difference squared between the outages when the ICI is known and the estimation of the outages when the ICI is unknown. The smaller 61 and 62 are, the more accurate the estimates $\bar{R}_{1, e}$ and $\bar{R}_{2, e}$ become. In fact, when $\delta_{1} \rightarrow 0$, and $\delta_{2} \rightarrow 0$, $\bar{R}_{1, e} \rightarrow \bar{R}_{1}$ and $\bar{R}_{2, e} \rightarrow \bar{R}_{2}$.

Now, if $\delta_{1}<\epsilon$ and $\delta_{2}<\epsilon$ hold, the constants $\gamma_{I 1}$ and $\gamma_{I 2}$ that maximize the estimated BS-U1 and BS-U2 throughput region, defined in 59 and (60), can be found from the following maximization problem

$$
\begin{aligned}
\underset{\gamma_{I 1}, \gamma_{I 2} \cdot}{\operatorname{aaximize}:} & \lim _{T \rightarrow \infty} \frac{1}{T} \sum_{t=1}^{T} \mu R_{1}^{m^{*}} q_{1}^{m^{*}}(t) O_{1, e}^{m^{*}}(t) \\
+ & \lim _{T \rightarrow \infty} \frac{1}{T} \sum_{t=1}^{T}(1-\mu) R_{2}^{l^{*}} q_{2}^{l^{*}}(t) O_{2, e}^{l^{*}}(t)
\end{aligned}
$$

Subject to :

$$
\begin{aligned}
& \mathrm{C} 1: \lim _{T \rightarrow \infty} \frac{1}{T} \sum_{t=1}^{T} q_{1}^{m^{*}}(t)\left(O_{1}^{m^{*}}(t)-O_{1, e}^{m^{*}}(t)\right)^{2} \leq \epsilon, \\
& \mathrm{C} 2: \lim _{T \rightarrow \infty} \frac{1}{T} \sum_{t=1}^{T} q_{2}^{l^{*}}(t)\left(O_{2}^{l^{*}}(t)-O_{2, e}^{l^{*}}(t)\right)^{2} \leq \epsilon,
\end{aligned}
$$

where $m^{*}=\arg \max _{m}\left\{R_{1}^{m} O_{1, e}^{m}(t)\right\}$ and $l^{*}=$ $\arg \max _{l}\left\{R_{2}^{l} O_{2, e}^{l}(t)\right\}$.

By applying the Lagrangian function on 63, we obtain

$$
\begin{aligned}
\mathcal{L}= & -\lim _{T \rightarrow \infty} \frac{1}{T} \sum_{t=1}^{T} \mu R_{1}^{m^{*}} q_{1}^{m^{*}}(t) O_{1, e}^{m^{*}}(t) \\
& -\lim _{T \rightarrow \infty} \frac{1}{T} \sum_{t=1}^{T}(1-\mu) R_{2}^{l^{*}} q_{2}^{l^{*}}(t) O_{2, e}^{l^{*}}(t) \\
& +\lim _{T \rightarrow \infty} \frac{1}{T} \sum_{t=1}^{T} \chi_{1} q_{1}^{m^{*}}(t)\left(O_{1}^{m^{*}}(t)-O_{1, e}^{m^{*}}(t)\right)^{2} \\
& +\lim _{T \rightarrow \infty} \frac{1}{T} \sum_{t=1}^{T} \chi_{2} q_{2}^{l^{*}}(t)\left(O_{2}^{l^{*}}(t)-O_{2, e}^{l^{*}}(t)\right)^{2},
\end{aligned}
$$

where $\chi_{1} \geq 0$ and $\chi_{2} \geq 0$ are the Lagrangian multipliers, found such that $\mathrm{C} 1$ and $\mathrm{C} 2$ in 63 hold. By differentiating $\mathcal{L}$ in 64 with respect to $\gamma_{I 1}$ and $\gamma_{I 2}$, and equivalenting the results to zero, we obtain

$$
\begin{aligned}
& \lim _{T \rightarrow \infty} \frac{1}{T} \sum_{t=1}^{T}\left[\frac{-P_{1} \gamma_{1}(t) \delta_{1}^{m^{*}}(t) q_{1}^{m^{*}}(t)}{\ln (2)\left(1+\gamma_{I 1}+P_{1} \gamma_{1}(t)\right)\left(1+\gamma_{I 1}\right)}\right. \\
& \left.\times\left(-\mu R_{1}^{m^{*}}+2 \chi_{1}\left[O_{1, e}^{m^{*}}(t)-O_{1}^{m^{*}}(t)\right]\right)\right]=0, \\
& \lim _{T \rightarrow \infty} \frac{1}{T} \sum_{t=1}^{T}\left[\frac{-P_{2} \gamma_{2}(t) \delta_{2}^{l^{*}}(t) q_{2}^{l^{*}}(t)}{\ln (2)\left(1+\gamma_{I 2}+P_{2} \gamma_{2}(t)\right)\left(1+\gamma_{I 2}\right)}\right. \\
& \left.\quad \times\left(-(1-\mu) R_{2}^{l^{*}}+2 \chi_{2}\left[O_{2, e}^{l^{*}}(t)-O_{2}^{l^{*}}(t)\right]\right)\right]=0 .
\end{aligned}
$$

Due to the law of large numbers, 65 and 66 can be written as

$$
\begin{aligned}
& E\left[\frac{-P_{1} \gamma_{1}(t) \delta_{1}^{m^{*}}(t) q_{1}^{m^{*}}(t)}{\ln (2)\left(1+\gamma_{I 1}+P_{1} \gamma_{1}(t)\right)\left(1+\gamma_{I 1}\right)}\right. \\
& \left.\quad \times\left(-\mu R_{1}^{m^{*}}+2 \chi_{1}\left[O_{1, e}^{m^{*}}(t)-O_{1}^{m^{*}}(t)\right]\right)\right]=0,
\end{aligned}
$$




$$
\begin{aligned}
& E\left[\frac{-P_{2} \gamma_{2}(t) \delta_{2}^{l^{*}}(t) q_{2}^{l^{*}}(t)}{\ln (2)\left(1+\gamma_{I 2}+P_{2} \gamma_{2}(t)\right)\left(1+\gamma_{I 2}\right)}\right. \\
& \left.\quad \times\left(-(1-\mu) R_{2}^{l^{*}}+2 \chi_{2}\left[O_{2, e}^{l^{*}}(t)-O_{2}^{l^{*}}(t)\right]\right)\right]=0 .
\end{aligned}
$$

Calculating the constants $\gamma_{I 1}$ and $\gamma_{I 2}$ from 67) and 68 requires the derivation of the above expectations. Instead, we use a more practical approach, where the constants $\gamma_{I 1}$ and $\gamma_{I 2}$ are estimated as $\gamma_{I 1}^{e}(t)$ and $\gamma_{I 2}^{e}(t)$ in time slot $t$. To this end, we apply the gradient descent method [32] on (65) and (66) to obtain $\gamma_{I 1}^{e}(t)$ and $\gamma_{I 2}^{e}(t)$ as in (28) and (29), where $\delta_{k}(t)$ for $k \in\{1,2\}$ is an adaptive step size which controls the speed of convergence of $\gamma_{I k}^{e}(t)$ to $\gamma_{I k}$, for $k \in\{1,2\}$, which can be some properly chosen monotonically decaying function of $t$ with $\delta_{k}(1)<1$. Note that $\lim _{t \rightarrow \infty} \gamma_{I 1}^{e}(t)=\gamma_{I 1}$ and $\lim _{t \rightarrow \infty} \gamma_{I 2}^{e}(t)=\gamma_{I 2}$. This completes the proof.

\section{Proof of Theorem 3}

In time slot $t$, an outage occurs if the BS-U1 channel is selected for transmission and the BS-U1 channel is too weak to support the rate $R_{0}$, i.e., $q_{1}^{1}(t)=1$ and $O_{1}^{1}(t)=0$, or if the BS-U2 channel is selected to transmission and the BS-U2 channel is too weak to support the rate $R_{0}$, i.e., $q_{2}^{1}(t)=1$ and $O_{2}^{1}(t)=0$, or if both the BS-U1 and BS-U2 channels are not selected for transmission in time slot $t$, i.e., if $q_{1}^{1}(t)=q_{2}^{1}(t)=0$, since in that case the time slot $t$ is wasted. Assuming that $\gamma_{I 1}^{e}(t)$ and $\gamma_{I 2}^{e}(t)$ have converged to their steady states given by $\lim _{t \rightarrow \infty} \gamma_{I 1}^{e}(t)=\gamma_{I 1}$ and $\lim _{t \rightarrow \infty} \gamma_{I 2}^{e}(t)=\gamma_{I 2}$, the outage probability, $P_{\text {out }}$, can be found as

$$
\begin{gathered}
P_{\text {out }}=\operatorname{Pr}\left\{\left[q_{1}^{1}(t)=1 \text { AND } O_{1}^{1}(t)=0\right]\right. \\
\text { OR }\left[q_{2}^{1}(t)=1 \text { AND } O_{2}^{1}(t)=0\right] \\
\text { OR } \left.\left[q_{1}^{1}(t)=q_{2}^{1}(t)=0\right]\right\} \\
\stackrel{(a)}{=} \operatorname{Pr}\left\{q_{1}^{1}(t)=1 \text { AND } O_{1}^{1}(t)=0\right\} \\
+\operatorname{Pr}\left\{q_{2}^{1}(t)=1 \text { AND } O_{2}^{1}(t)=0\right\} \\
+\operatorname{Pr}\left\{q_{1}^{1}(t)=q_{2}^{1}(t)=0\right\},
\end{gathered}
$$

where $(a)$ follows since the events $q_{1}^{1}(t)=1$ and $q_{1}^{1}(t)=0$, and also the events $q_{2}^{1}(t)=1$ and $q_{2}^{1}(t)=0$ are mutually exclusive.

We divide 699 into three events; BS-U1 communication event, $\left[q_{1}^{1}(t)=1\right.$ AND $\left.O_{1}^{1}(t)=0\right]$, BS-U2 communication event $\left[q_{2}^{1}(t)=1\right.$ AND $\left.O_{2}^{1}(t)=0\right]$, and the silent event $\left[q_{1}^{1}(t)=q_{2}^{1}(t)=0\right]$. In the following, we calculate the probability of these three events.

For the BS-U1 communication event, we have $q_{1}^{1}(t)=1$ when either of the two following events occur

- $O_{1, e}^{1}(t)=1$ and $O_{2, e}^{1}(t)=0$. This event occurs when $\frac{\gamma_{1}(t)}{1+\gamma_{I 1}} \geq \gamma_{\text {th }}$ and $\frac{\gamma_{2}(t)}{1+\gamma_{I 2}}<\gamma_{\text {th }}$, where $\gamma_{\text {th }}=\frac{2^{R_{0}}-1}{P}$.

- $O_{1, e}^{1}(t)=O_{2, e}^{1}(t)=1$ and $\gamma_{U}^{e}(t) \geq \gamma_{D}^{e}(t)$. This event occurs when $\frac{\gamma_{1}(t)}{1+\gamma_{I 1}}>\gamma_{\mathrm{th}}, \frac{\gamma_{2}(t)}{1+\gamma_{I 2}}>\gamma_{\mathrm{th}}$, and $\frac{\gamma_{1}(t)}{1+\gamma_{I 1}} \geq \frac{\gamma_{2}(t)}{1+\gamma_{I 2}}$.
On the other hand, the event $O_{1}^{1}(t)=0$ occurs with the following probability

$$
\operatorname{Pr}\left\{O_{1}^{1}(t)=0\right\}=\operatorname{Pr}\left(\frac{\gamma_{1}(t)}{1+\gamma_{I 1}(t)}<\gamma_{\mathrm{th}}\right),
$$

where $\gamma_{I 1}(t)$ is the sum of $K$ identical and independent random variables with mean (identical mean is assumed for simplicity) $\Omega_{I}$, which has the following PDF

$$
f_{\gamma_{I 1}}(z)=\frac{z^{K-1} e^{-\frac{z}{\Omega_{I}}}}{\Omega_{I}^{K}(K-1) !}
$$

and the following cumulative distribution function (CDF)

$$
F_{\gamma_{I 1}}(z)=1-\sum_{n=0}^{K-1} \frac{1}{n !} e^{-\frac{z}{\Omega_{I}}}\left(\frac{z}{\Omega_{I}}\right)^{n} .
$$

In addition, the variables $\gamma_{1}(t)$ and $\gamma_{2}(t)$ have i.i.d. exponential distributions with PDFs, $f_{\gamma_{1}}\left(\gamma_{1}\right)$ and $f_{\gamma_{2}}\left(\gamma_{2}\right)$, respectively, that have mean $\Omega_{0}=E\left\{\frac{\left|h_{1}(t)\right|^{2}}{\sigma_{1}^{2}}\right\}=E\left\{\frac{\left|h_{2}(t)\right|^{2}}{\sigma_{2}^{2}}\right\}$.

Using the above, we can rewrite $\operatorname{Pr}\left\{q_{1}^{1}(t)=\right.$ 1 AND $\left.O_{1}^{1}(t)=0\right\}$ in an integral form as

$$
\begin{aligned}
& \operatorname{Pr}\left\{q_{1}^{1}(t)=1 \text { AND } O_{1}^{1}(t)=0\right\} \\
& =\int_{\gamma_{\mathrm{th}}\left(1+\gamma_{I 2}\right)}^{\infty} \int_{0}^{\gamma_{\mathrm{th}}\left(1+\gamma_{I 1}\right)} \operatorname{Pr}\left(Z>\frac{\gamma_{1}}{\gamma_{\mathrm{th}}}-1\right) f_{\gamma_{1}}\left(\gamma_{1}\right) f_{\gamma_{2}}\left(\gamma_{2}\right) d \gamma_{2} d \gamma_{1} \\
& +\int_{\gamma_{\mathrm{th}}\left(1+\gamma_{I 2}\right)}^{\infty} \int_{\gamma_{2}\left(\frac{1+\gamma_{I 1}}{1+\gamma_{I 2}}\right)}^{\infty} \operatorname{Pr}\left(Z>\frac{\gamma_{1}}{\gamma_{\mathrm{th}}}-1\right) f_{\gamma_{1}}\left(\gamma_{1}\right) f_{\gamma_{2}}\left(\gamma_{2}\right) d \gamma_{1} d \gamma_{2} \\
& \stackrel{(a)}{=} \int_{\gamma_{\mathrm{th}}\left(1+\gamma_{I 1}\right)}^{\infty} \int_{0}^{\gamma_{1}\left(\frac{1+\gamma_{I 2}}{1+\gamma_{\gamma_{1}}}\right)} \sum_{n=0}^{K-1} \frac{1}{n !} e^{-\left(\frac{\left[\frac{\gamma_{1}}{\gamma_{\mathrm{th}}}-1\right]}{\Omega_{I}}\right)}\left(\frac{\left[\frac{\gamma_{1}}{\gamma_{\mathrm{th}}}-1\right]}{\Omega_{I}}\right)^{n}
\end{aligned}
$$

where (a) follows from 72 . Now, performing the integration with respect to $\gamma_{2}$, we obtain

$$
\begin{aligned}
& \operatorname{Pr}\left\{q_{1}^{1}(t)=1 \text { AND } O_{1}^{1}(t)=0\right\} \\
& =\frac{\gamma_{\mathrm{th}} \Omega_{I} e^{-\left(\frac{\gamma_{\mathrm{th}}}{\Omega_{0}}\right)}}{\Omega_{0}} \int_{\frac{\gamma_{I 1}}{\Omega_{I}}}^{\infty} \sum_{n=0}^{K-1} \frac{1}{n !} e^{-U^{\prime}}\left(U^{\prime}\right)^{n} e^{-\left(\frac{\gamma_{\mathrm{th}} \Omega_{I} U^{\prime}}{\Omega_{0}}\right)} \\
& \times\left(1-e^{-\left[\left(\frac{\gamma_{\mathrm{th}}}{\Omega_{0}}\right)\left(1+\Omega_{I} U^{\prime}\right)\left(\frac{1+\gamma_{I 2}}{1+\gamma_{I 1}}\right)\right]}\right) d U^{\prime},
\end{aligned}
$$

where $U^{\prime}=\frac{\left(\frac{\gamma_{1}}{\gamma_{\mathrm{th}}}-1\right)}{\Omega_{I}}$. In (74), when $P \rightarrow \infty$, and consequently $\gamma_{\text {th }} \rightarrow 0$, we can approximate $\left(1-e^{-\left[\left(\frac{\gamma_{\text {th }}}{\Omega_{0}}\right)\left(1+\Omega_{I} U^{\prime}\right)\left(\frac{1+\gamma_{I 2}}{1+\gamma_{I 1}}\right)\right]}\right)$ by $\left(\left[\left(\frac{\gamma_{\text {th }}}{\Omega_{0}}\right)\left(1+\Omega_{I} U^{\prime}\right)\left(\frac{1+\gamma_{I 2}}{1+\gamma_{I 1}}\right)\right]\right)$, $e^{-\left(\frac{\gamma_{\text {th }}}{\Omega_{0}}\right)}$ by 1 , and $e^{-\left(\frac{\gamma_{\mathrm{th}} \Omega_{I} U^{\prime}}{\Omega_{0}}\right)}$ by 1 . As a result, (74) can be 
rewritten as

$$
\begin{aligned}
& \operatorname{Pr}\left\{q_{1}^{1}(t)=1 \text { AND } O_{1}^{1}(t)=0\right\} \\
& \rightarrow \frac{\gamma_{\mathrm{th}}^{2} \Omega_{I}}{\Omega_{0}^{2}} \times \frac{1+\gamma_{I} 2}{1+\gamma_{I}}\left[\sum_{n=0}^{K-1} \frac{1}{n !} \int_{\frac{\gamma_{I 1}}{\Omega_{I}}}^{\infty} e^{-U^{\prime}}\left(U^{\prime}\right)^{n} d U^{\prime}\right. \\
& \left.+\Omega_{I} \sum_{n=0}^{K-1} \frac{1}{n !} \int_{\frac{\gamma_{I 1}}{\Omega_{I}}}^{\infty} e^{-U^{\prime}}\left(U^{\prime}\right)^{n+1} d U^{\prime}\right] \text { as } P \rightarrow \infty .
\end{aligned}
$$

By calculating the integrals and the summations in (75), we obtain

$$
\operatorname{Pr}\left\{q_{1}^{1}(t)=1 \text { AND } O_{1}^{1}(t)=0\right\} \rightarrow \frac{\gamma_{\mathrm{th}}^{2} \hat{\Omega}_{I 1}}{\Omega_{0}^{2}} \text { as } P \rightarrow \infty,
$$

where $\hat{\Omega}_{I 1}$ is given in 38 .

For the BS-U2 communication event, we obtain that $q_{2}^{1}(t)=$ 1 if $O_{2, e}^{1}(t)=1$ and $O_{1, e}^{1}(t)=0$, or $O_{2, e}^{1}(t)=O_{1, e}^{1}(t)=1$ and $\frac{\gamma_{1}(t)}{1+\gamma_{I 1}}<\frac{\gamma_{2}(t)}{1+\gamma_{I 2}}$. The event $O_{2, e}^{1}(t)=1$ and $O_{1, e}^{1}(t)=0$ occurs when $\frac{\gamma_{2}(t)}{1+\gamma_{I 2}}>\gamma_{\mathrm{th}}$ and $\frac{\gamma_{1}(t)}{1+\gamma_{I 1}}<\gamma_{\mathrm{th}}$. The event $O_{2, e}^{1}(t)=O_{1, e}^{1}(t)=1$ and $\frac{\gamma_{1}(t)}{1+\gamma_{I 1}}<\frac{\gamma_{2}(t)}{1+\gamma_{I 2}}$ occurs when $\frac{\gamma_{2}(t)}{1+\gamma_{I 2}}>\gamma_{\mathrm{th}}, \frac{\gamma_{1}(t)}{1+\gamma_{I 1}}>\gamma_{\mathrm{th}}$, and $\frac{\gamma_{1}(t)}{1+\gamma_{I 1}}<\frac{\gamma_{2}(t)}{1+\gamma_{I 2}}$. Using this, we can derive $\operatorname{Pr}\left\{q_{2}^{1}(t)=1\right.$ AND $\left.O_{2}^{1}(t)=0\right\}$ with a similar approach as the calculation of $\operatorname{Pr}\left\{q_{1}^{1}(t)=1\right.$ AND $\left.O_{1}^{1}(t)=0\right\}$, which results in

$$
\operatorname{Pr}\left\{q_{2}^{1}(t)=1 \text { AND } O_{2}^{1}(t)=0\right\} \rightarrow \frac{\gamma_{\mathrm{th}}^{2} \hat{\Omega}_{I 2}}{\Omega_{0}^{2}} \text { as } P \rightarrow \infty,
$$

where $\hat{\Omega}_{I 2}$ is given in 39 .

Finally, for the silent event, we obtain that $q_{1}^{1}(t)=q_{2}^{1}(t)=$ 0 occurs iff $O_{1, e}^{1}(t)=O_{2, e}^{1}(t)=0$ holds, which occurs when $\frac{\gamma_{2}(t)}{1+\gamma_{I 2}}<\gamma_{\mathrm{th}}$, and $\frac{\gamma_{1}(t)}{1+\gamma_{I 1}}<\gamma_{\mathrm{th}}$. The probability of the silent event can be calculated by

$$
\begin{aligned}
& \operatorname{Pr}\left\{q_{1}^{1}(t)=q_{2}^{1}(t)=0\right\} \\
& =\frac{1}{\Omega_{0}^{2}} \int_{0}^{\gamma_{\mathrm{th}}\left(1+\gamma_{I 2}\right) \gamma_{\mathrm{th}}\left(1+\gamma_{I 1}\right)} \int_{0}^{-\left(\frac{\gamma_{1}}{\Omega_{0}}\right)} e^{-\left(\frac{\gamma_{2}}{\Omega_{0}}\right)} d \gamma_{1} d \gamma_{2} .
\end{aligned}
$$

The expression in (78) for $P \rightarrow \infty$, and consequently $\gamma_{\mathrm{th}} \rightarrow$ 0 , converges to

$$
\operatorname{Pr}\left\{q_{1}^{1}(t)=q_{2}^{1}(t)=0\right\} \rightarrow \frac{\gamma_{\mathrm{th}}^{2}}{\Omega_{0}^{2}} \hat{\Omega}_{I S} \text { as } P \rightarrow \infty,
$$

where $\hat{\Omega}_{I S}$ is given in 40).

Now, by adding (76), (77), and (79), we obtain the asymptotic outage probability as in (37). This completes the proof.

\section{REFERENCES}

[1] M. M. Razlighi, N. Zlatanov, and P. Popovski, "On distributed dynamictdd schemes for base stations with decoupled uplink-downlink transmissions," in 2018 IEEE International Conference on Communications Workshops (ICC Workshops), May 2018, pp. 1-6.

[2] H. Holma and A. Toskala, LTE for UMTS: Evolution to LTE-Advanced. Wiley, 2011.

[3] "Further advancements for E-UTRA physical layer aspects," Technical Report, document TR 36.814, Release 9, v9.0.0, 3GPP, Mar. 2010.
[4] A. K. Gupta, M. N. Kulkarni, E. Visotsky, F. W. Vook, A. Ghosh, J. G. Andrews, and R. W. Heath, "Rate analysis and feasibility of dynamic tdd in 5g cellular systems," in 2016 IEEE International Conference on Communications (ICC), May 2016, pp. 1-6.

[5] Z. Shen, A. Khoryaev, E. Eriksson, and X. Pan, "Dynamic uplinkdownlink configuration and interference management in TD-LTE," IEEE Communications Magazine, vol. 50, no. 11, pp. 51-59, November 2012.

[6] S. Adireddy and L. Tong, "Exploiting decentralized channel state information for random access," IEEE Transactions on Information Theory, vol. 51, no. 2, pp. 537-561, Feb 2005.

[7] S. Lagen, A. Agustin, and J. Vidal, "Joint user scheduling, precoder design, and transmit direction selection in mimo tdd small cell networks," IEEE Transactions on Wireless Communications, vol. 16, no. 4, pp. 2434-2449, April 2017.

[8] K. Lee, Y. Park, M. Na, H. Wang, and D. Hong, "Aligned reverse frame structure for interference mitigation in dynamic tdd systems," IEEE Transactions on Wireless Communications, vol. 16, no. 10, pp. 6967-6978, Oct 2017.

[9] H. Haas and S. McLaughlin, "A dynamic channel assignment algorithm for a hybrid tdma/cdma-tdd interface using the novel ts-opposing technique," IEEE Journal on Selected Areas in Communications, vol. 19, no. 10, pp. 1831-1846, Oct 2001.

[10] C. Yoon and D. H. Cho, "Energy efficient beamforming and power allocation in dynamic tdd based c-ran system," IEEE Communications Letters, vol. 19, no. 10, pp. 1806-1809, Oct 2015.

[11] P. Popovski, O. Simeone, J. J. Nielsen, and C. Stefanovic, "Interference spins: Scheduling of multiple interfering two-way wireless links," IEEE Communications Letters, vol. 19, no. 3, pp. 387-390, March 2015.

[12] K. Sivarajah and H. S. Al-Raweshidy, "Dynamic channel allocation for ongoing calls in utra tdd system," Electronics Letters, vol. 40, no. 19, pp. 1197-1198, Sept 2004.

[13] C. H. Chiang, W. Liao, T. Liu, I. K. Chan, and H. L. Chao, "Adaptive downlink and uplink channel split ratio determination for tcp-based best effort traffic in tdd-based wimax networks," IEEE Journal on Selected Areas in Communications, vol. 27, no. 2, pp. 182-190, February 2009.

[14] J. Huang, K. Qi, Z. Xu, and C. Yang, "Hybrid full and half duplex networking," in 2016 IEEE/CIC International Conference on Communications in China (ICCC), July 2016, pp. 1-6.

[15] J. Liu, S. Han, W. Liu, and C. Yang, "The value of full-duplex for cellular networks: A hybrid duplex based study," IEEE Transactions on Communications, vol. PP, no. 99, pp. 1-1, 2017.

[16] S. Goyal, P. Liu, and S. S. Panwar, "User selection and power allocation in full-duplex multicell networks," IEEE Transactions on Vehicular Technology, vol. 66, no. 3, pp. 2408-2422, March 2017.

[17] K. Smiljkovikj, P. Popovski, and L. Gavrilovska, "Analysis of the decoupled access for downlink and uplink in wireless heterogeneous networks," IEEE Wireless Communications Letters, vol. 4, no. 2, pp. 173-176, April 2015.

[18] A. A. Dowhuszko, O. Tirkkonen, J. Karjalainen, T. Henttonen, and J. Pirskanen, "A decentralized cooperative uplink/downlink adaptation scheme for tdd small cell networks," in 2013 IEEE 24th Annual International Symposium on Personal, Indoor, and Mobile Radio Communications (PIMRC), Sept 2013, pp. 1682-1687.

[19] B. Yu, L. Yang, H. Ishii, and S. Mukherjee, "Dynamic TDD support in macrocell-assisted small cell architecture," IEEE Journal on Selected Areas in Communications, vol. 33, no. 6, pp. 1201-1213, June 2015.

[20] Key Technologies for $5 G$ Wireless Systems. Cambridge University Press, 2017.

[21] R. Veronesi, V. Tralli, J. Zander, and M. Zorzi, "Distributed dynamic resource allocation for multicell sdma packet access net," IEEE Transactions on Wireless Communications, vol. 5, no. 10, pp. 2772-2783, Oct 2006.

[22] I. Spyropoulos and J. R. Zeidler, "Supporting asymmetric traffic in a tdd/cdma cellular network via interference-aware dynamic channel allocation and space-time lmmse joint detection," IEEE Transactions on Vehicular Technology, vol. 58, no. 2, pp. 744-759, Feb 2009.

[23] Y. Yu and G. B. Giannakis, "Opportunistic medium access for wireless networking adapted to decentralized csi," IEEE Transactions on Wireless Communications, vol. 5, no. 6, pp. 1445-1455, June 2006.

[24] V. Venkatasubramanian, M. Hesse, P. Marsch, and M. Maternia, "On the performance gain of flexible ul/dl tdd with centralized and decentralized resource allocation in dense 5g deployments," in 2014 IEEE 25th Annual International Symposium on Personal, Indoor, and Mobile Radio Communication (PIMRC), Sept 2014, pp. 1840-1845.

[25] R. Wang and V. K. N. Lau, "Robust optimal cross-layer designs for tdd-ofdma systems with imperfect csit and unknown interference: Statespace approach based on 1-bit ack/nak feedbacks," IEEE Transactions on Communications, vol. 56, no. 5, pp. 754-761, May 2008. 
[26] S. Sekander, H. Tabassum, and E. Hossain, "Decoupled uplink-downlink user association in multi-tier full-duplex cellular networks: A two-sided matching game," IEEE Transactions on Mobile Computing, vol. PP, no. 99, pp. 1-1, 2016.

[27] F. Boccardi, J. Andrews, H. Elshaer, M. Dohler, S. Parkvall, P. Popovski, and S. Singh, "Why to decouple the uplink and downlink in cellular networks and how to do it," IEEE Communications Magazine, vol. 54 no. 3, pp. 110-117, March 2016.

[28] J. N. Laneman, D. N. C. Tse, and G. W. Wornell, "Cooperative diversity in wireless networks: Efficient protocols and outage behavior," IEEE Transactions on Information Theory, vol. 50, no. 12, pp. 3062-3080, Dec 2004

[29] D. Nguyen, L. N. Tran, P. Pirinen, and M. Latva-aho, "On the spectral efficiency of full-duplex small cell wireless systems," IEEE Transactions on Wireless Communications, vol. 13, no. 9, pp. 4896-4910, Sept 2014

[30] S. Goyal, P. Liu, S. S. Panwar, R. A. Difazio, R. Yang, and E. Bala, "Full duplex cellular systems: will doubling interference prevent doubling capacity?" IEEE Communications Magazine, vol. 53, no. 5, pp. 121127, May 2015.

[31] Y. Sun, D. W. K. Ng, J. Zhu, and R. Schober, "Multi-objective optimization for robust power efficient and secure full-duplex wireless communication systems," IEEE Transactions on Wireless Communications, vol. 15, no. 8, pp. 5511-5526, Aug 2016.

[32] S. Boyd and L. Vandenberghe, Convex Optimization. Cambridge University Press, 2004. 